Article

\title{
Computational Colour Matching of Laminated Photovoltaic Modules for Building Envelopes
}

\author{
Roland Schregle* (iD, Marek Krehel (i) and Stephen Wittkopf \\ Competence Centre Building Envelopes, Lucerne University of Applied Sciences and Arts, Technikumstrasse 21, \\ CH-6048 Horw, Switzerland; marek.krehel@hslu.ch (M.K.); stephen.wittkopf@hslu.ch (S.W.) \\ * Correspondence: roland.schregle@hslu.ch; Tel.: +41-41-349-3626
}

Received: 8 June 2017; Accepted: 11 August 2017; Published: 18 August 2017

\begin{abstract}
The widespread adoption of photovoltaics (PV) in architecture as a source of renewable energy is often limited due to poor visual acceptance. We propose the use of coloured frontglass manufactured by digital ceramic printing to cover the PV cells, thus concealing the latter while admitting sufficient light to produce electricity. The apparent colour of the PV laminate is a combination of the transparent colour on glass and the colour of the PV cells, which is difficult to predict. In this paper we compare 1400 unknown PV laminate colours with 320 known façade colours from the NCS Exterior Set to find potential matches. We use an image-based approach in which photographs are compared computationally by CIE $\Delta E$ colour difference. For a barely discernible $\Delta E<3.5$, this yielded 20 unique matches between the candidate and target sample sets, increasing to 74 matches for an obvious difference of $\Delta E \leq 10$. A subset of these computed matches was then visually confirmed by untrained volunteer observers, with average deviations of $\Delta E \approx 5$ between computed and visual matches, and a similar tolerance for the visual matches based on standard deviation. We conclude that our image-based computational approach enables a rapid and comprehensive matching of large sets of printed glass colours, which would be impractical with human subjects, yet delivers matches consistent with the latter's observations.
\end{abstract}

Keywords: building integrated photovoltaics; colour perception; colour difference

\section{Introduction}

The demand for renewable energy is increasingly addressed through the use of photovoltaic (PV) panels. One of the possible installation options for PV is building envelopes. PV installed on building envelopes (building integrated photovoltaics, or BIPV) has increasingly attracted the attention of architects, urban planners and engineers. Unfortunately, the installation of BIPV is not always accepted due to compromised visual appeal of the building, particularly because dark PV panels can significantly influence the original appearance of cultural and natural heritage [1]. Additionally, $\mathrm{PV}$ can increase glare probability, thus hindering the application of BIPV.

In order to overcome this issue and promote the application of BIPV we propose to modify the appearance of PV modules, such that they would not only generate electrical energy but also carry visual appeal and blend in with their surroundings. Recent studies have demonstrated the use of coloured PV for better visual integration [2,3]. We developed a similar approach by colouring the frontglass with which the PV is laminated [4]. Since PV generates a significant amount of its energy in the infrared (IR) range [5], the front glass can be coated with paint as long as it is transparent to IR. Our approach therefore benefits from the partial transparency in visible light. However, not all printable colours are well suited since certain dyes are more likely to absorb IR radiation and thus have to be chosen carefully.

In this paper, we focus on modifying the front glass using digital ceramic printing, which is a standard procedure in the glass industry and thus cost effective [6]. However, printing glass for 
PV modules has particular requirements, as the printed glass must be translucent for the PV cells to receive enough light to perform. The apparent colour of such a PV module is a combination of the translucent colour of the front glass and the transmitted opaque colours of the PV cells, multiwires and backsheet. Therefore when a front glass is printed in a desired translucent colour, the apparent colour in combination with the PV will be considerably different and difficult to predict.

While the science behind the electrical parameters of the PV modules is well established and documented [7], the perceived colour resulting from the blend of different layers is not. Such knowledge is essential to effectively integrate coloured BIPV onto buildings and predict its visual impact on the building. Architects and planners welcome this development in general but are accustomed to specifying colours using prevailing colour nomenclature such as RAL or Natural Colour System (NCS). Therefore in this article we propose a tool that matches desired NCS colours [8] to apparent colours of printed front glass combined with PV. Moreover, we present a metric and visual evaluation of our approach.

\section{Materials and Methods}

The developed method relies on a best match approach, and is summarised in Figure 1. Given a set of unknown candidate colours printed on glass merged with a PV module, and a set of known target colours representative of building façades, we find a subset of closest matches. In the presence of discrepancies between the candidate and target colours, it is unrealistic to expect a large number of exact matches, and this is reflected in our methodology.

In the first phase of our method, we employ an objective approach that relies on a quantifiable metric. This entails an image-based matching of colour-corrected photographs (captures) of the candidate and target colours. Since multiple colours are captured simultaneously per image, the rationale of this approach lies in the higher throughput over a more laborious comparison involving individual colorimetry. We then find the closest matches computationally using the CIE $\Delta E$ colour difference metric.

The CIE (International Commission on Illumination) revised its colour difference metric over the years since its original formulation in 1976. Originally, $\Delta E_{1976}$ was simply defined as the Euclidean distance of two colours in the $L^{*} a^{*} b^{*}$ colour space. The more recent $\Delta E_{1994}$ formulation takes perceptual non-uniformities into account, since the human eye is not equally sensitive to all colours. It does this by transforming the colours into an $L^{*} C^{*} h^{*}$ space to compensate for differences in luminance, chroma, and hue along with associated application-specific weights $k_{L}, k_{C}$, and $k_{h}$. The latest formulation, $\Delta E_{2000}$, adds compensation for neutral colours and problematic blue hues [9]. CIE has obsoleted use of the earlier formulations, and unless noted otherwise, we use $\Delta E_{2000}$ with weights $k_{L}=k_{C}=k_{h}=1$ in this paper.

Once we have obtained the set of computed matches, we verify these results in a second phase of our method with a subjective approach that relies on visual matching. At this point we draw on volunteer observers to match a subset of the candidate and target colours by eye under controlled conditions independently of the computed matches.

In the final phase, we evaluate the correlation between the computed and visual matches, taking into account their respective tolerances. We consider the accuracy of our methodology to be adequate for our application, taking into account deviations in the image capture on the one hand, and the ability of casual observers to differentiate colours on the other. 


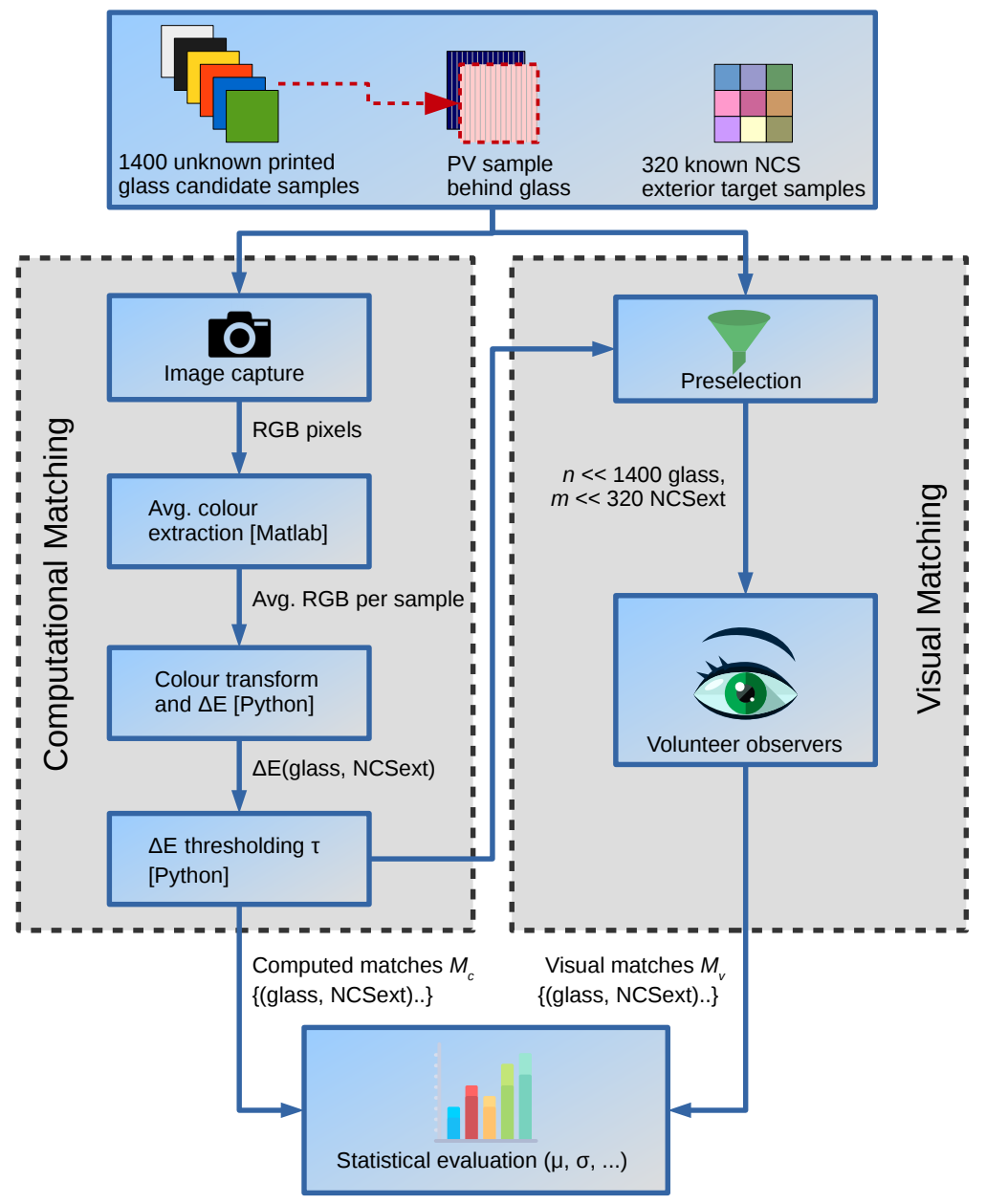

Figure 1. Overview of our coloured PV matching methodology. Using an image-based approach, we computationally match a subset of the 1400 unknown candidate colours on printed glass combined with PV to a subset of the 320 known target NCS exterior colours based on the pairwise $\Delta E$ colour difference. We then verify the set of computed matches $M_{\mathcal{C}}$ with a set of visual matches $M_{v}$ using volunteer observers, and evaluate them statistically.

\subsection{Material Selection}

The set of $70 \times 10 \times 2=1400$ unknown candidate colours printed on glass were selected in combination with a PV module. We refer to these as candidates as any of them may be selected for a match without explicit knowledge of their actual appearance combined with PV. These 1400 samples are comprised as follows:

- 70 colours from the Fabraum Stadt set [10] representing ca. $96 \%$ of exterior façades in the city of Zürich, with each colour contributing at least $0.2 \%$. These colours are expressed in NCS notation and comprise the only survey of façade colours to be undertaken in Switzerland at the time of publication.

- 10 print densities/opacities, corresponding to $10-100 \%$ ink coverage in $10 \%$ increments.

- 2 ink volumes, consisting of 10 and 20 picolitres (pl), applied in multiple printing passes.

The 70 candidate colours and their corresponding red-green-blue (RGB) print settings are listed in Table A1. The resulting colours may deviate depending on the printer calibration and glass manufacturing process, and can thus only be approximated. Since this uncertainty is compounded by blending the colours with PV, we simply treat them as unknowns. This is justified by our methodology, which aims to match any arbitrary colour based only on its appearance with PV. Once a particular colour is matched, it can be reproduced with the same printer calibration. 
7 glass panels were laid out, each bearing 10 distinct colours (columns) in 10 print densities (rows) each, which resulted in 100 colour samples per panel (see Figure 2). The print density per colour ranged from $10 \%$ to $100 \%$ of the printer's maximum coverage. Two sets of glass panels were printed with ink volumes of 10 and $20 \mathrm{pl}$ per drop, resulting in 14 panels.

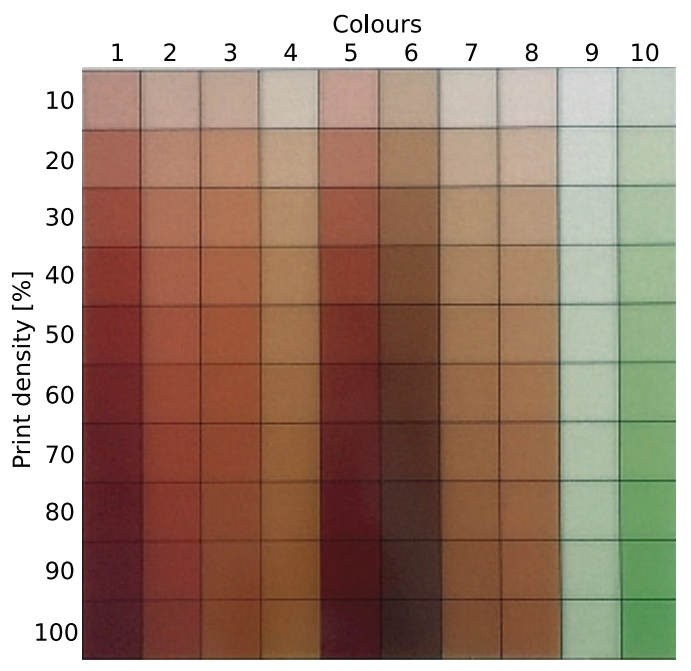

(a)

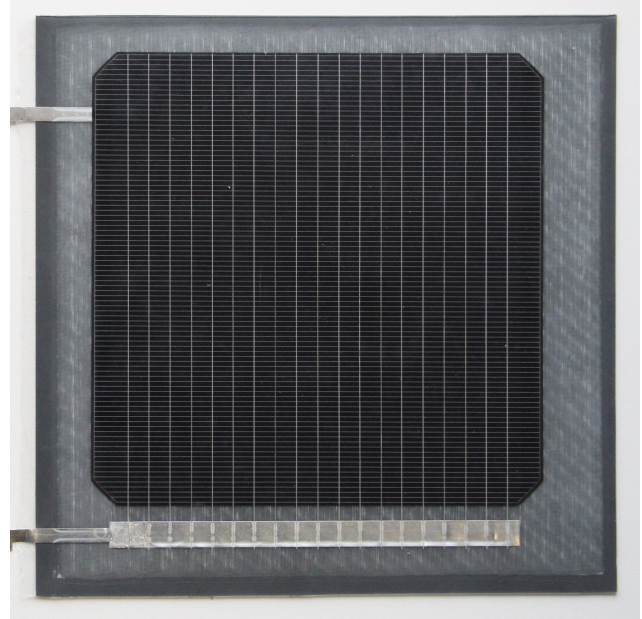

(b)

Figure 2. Sample printed glass panel with unknown candidate colours (a) and single-cell monocrystalline PV module (b) used for our matching method. Each column on the printed glass bears a unique colour, whose print density is varied per row in $10 \%$ increments. Two sets of glass panels were printed with ink volumes of 10 and 20 pl. Note this depiction is not to scale; the PV module is ca. $\frac{1}{4}$ the size of the glass panel (see Figure 3 for relative sizes).

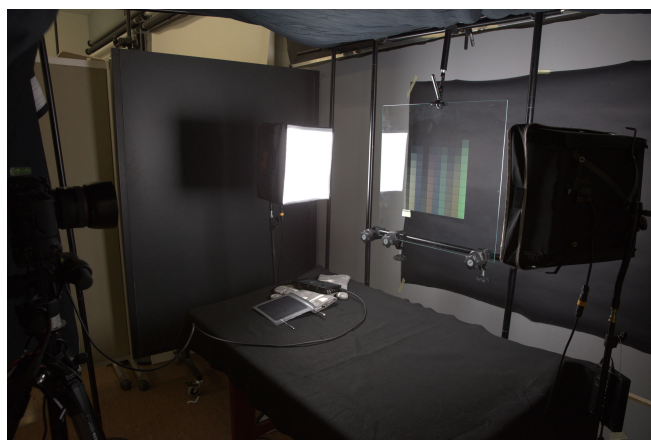

(a)

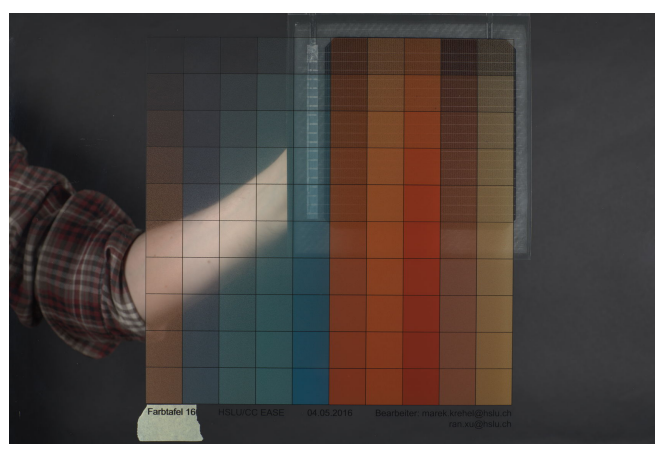

(b)

Figure 3. Image capture setup (a) for coloured glass samples. Uniform illumination is provided by two symmetrically placed LED studio lights. Each glass panel bearing 10 candidate colours in 10 print densities is mounted in a frame, allowing access to the back surface. The sample PV (b) is held against the glass panel's back surface in each of the four quadrants for a quartet of exposures.

Low-iron, high-clarity glass was used in the production of the printed glass samples. The samples were manufactured by Glas Trösch AG, Bützberg, Switzerland by means of a digital ceramic ink printing technique on a DipTech printer. The glass panels were of dimensions $60 \times 44 \mathrm{~cm}^{2}$, with the actual colour grid measuring ca. $30 \times 30 \mathrm{~cm}^{2}$.

The desired colours were achieved by mixing different amounts of base colours corresponding to those of the NCS system, i.e., black, white, yellow, green, blue and red. The printing pattern was applied in a frequency modulated grid by quasi-randomly positioning equal-sized dots to avoid undesirable Moiré effects. 
A single-cell monocrystalline PV module without glass cover having a rated efficiency of $18 \%$ was selected to be paired with the printed glass panels (see Figure 2). Compared to the predominantly blue polycrystalline modules also available on the PV market, the monocrystalline are dark grey and more neutral in hue, which increases the likelihood of a colour match. Due to the small size of the available PV sample (ca. $15 \times 15 \mathrm{~cm}$ ), we acquired four images per glass panel, with the PV covering one quadrant per exposure, corresponding to four subregions containing $5 \times 5$ sample colours (see Figure 3 ).

The set of 320 known target colours was selected from the NCS Exterior Set [11]. NCS recommends these colours for exterior applications on account of their perception in outdoor environments subject to daylight, typical urban surroundings, colour fastness, and conventions in contemporary and traditional colour design. The colours are characterised by low to moderate saturation (chromaticness $\leq 40 \%$ ), predominantly yellow-red hues, few pure blues, and no pure greens. Neutral greys with blackness up to $80 \%$ are included. Sample cards of the NCS exterior set were mounted on three panels in $10 \times 10$ groups, with the remaining $2 \times 10$ on a fourth panel (see Figure 4).
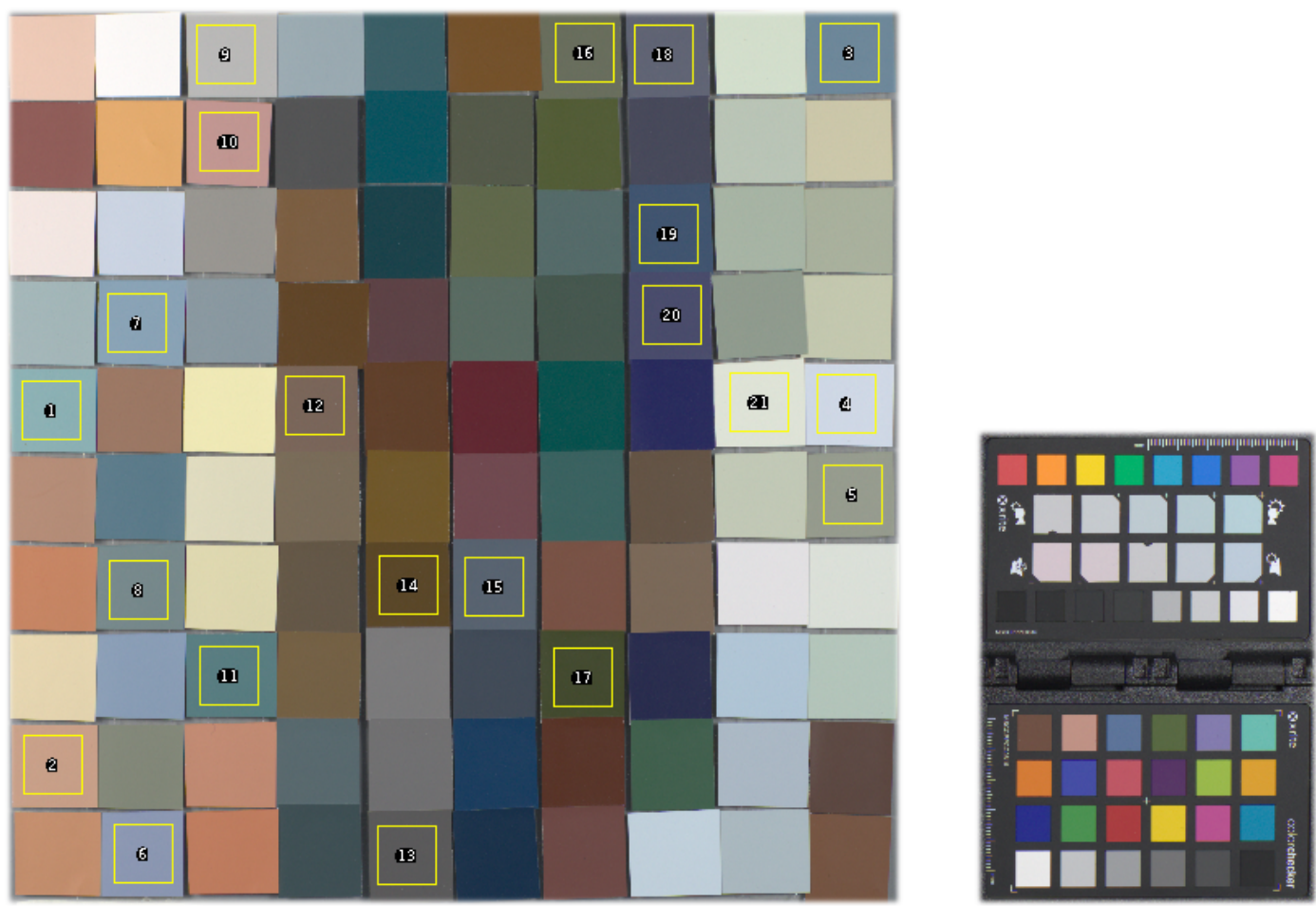

Figure 4. Colour calibrated image capture of 100 of the 320 NCS exterior set samples. The highlighted regions (a subset of the colours in Table A3) were compared for deviations against colorimetric readings to verify the accuracy of the image capture and colour calibration workflow in preparation for computational matching.

We refer to these colours as targets since we consider them as given in our methodology, and because their colorimetric reference values are known and thus quantifiable.

\subsection{Computational Matching}

Our computational matching involves four steps:

1. Photographic image capture of printed glass and NCS exterior set samples

2. Colour calibration of the captures

3. Average sample colour extraction from captures

4. Matching by pairwise colour difference $\Delta E$ of extracted sample colours.

These steps are detailed in the following sections. 


\subsubsection{Image Capture Procedure}

Photographs of the selected printed glass samples combined with PV, and of the NCS exterior samples, were taken under identical and reproducible conditions. A quartet of exposures was taken per printed glass panel, with the PV sample carefully placed by hand against the back surface so as to cover each quadrant containing $5 \times 5$ sample colours. To this end, the glass panels were mounted vertically in a frame to facilitate access to the front and back of the sample (see Figure 3).

We obtained RAW images from a Canon EOS 5D MkIV DSLR with $85 \mathrm{~mm}$ lens and fixed exposure time and aperture. A pair of Aladdin Bi-Flex LED studio lights served as illuminants, with a measured white point corresponding to a correlated colour temperature (CCT) of $6500 \mathrm{~K}$. The illuminants were positioned symmetrically at $45^{\circ}$ to the sample, and their individual brightness was adjusted for even illuminance in the corners according to a luxmeter. The acquisition setup was shielded from stray light and reflections with a black surround.

\subsubsection{Image Capture Colour Calibration}

A Colourchecker Passport was captured under the same conditions as the samples to perform colour calibration. An ICC (International Colour Consortium) profile was generated with the Argyll Colour Management System [12] from the RAW images using custom colorimetric reference values in the $L^{*} a^{*} b^{*}$ colour space. The latter were measured from the Colourchecker with a spectrophotometer (Colorlite model sph860), since the actual values have been found to deviate up to a $\Delta E$ of 0.97 from the manufacturer's reference data [13]. These measurements were performed under a D65 illuminant setting consistent with the $6500 \mathrm{~K} \mathrm{CCT}$ of the studio lighting used in the capture.

The ICC profile was then applied to all RAW captures in RawTherapee [14]. In addition, a fixed black point and exposure compensation was applied to match the minimum and maximum luminances of the Colourchecker's neutral patches. The calibrated images were then exported to the sRGB colour space in TIFF format for the colour extraction and computational matching described in Sections 2.2.4 and 2.2.5. Lens distortion was considered negligible for our application, and not corrected.

\subsubsection{Image Capture Verification}

We verified the accuracy of the colour correction as a prerequisite to our computational matching, since any errors in the captures will accumulate in the matching. This was done by comparing the 24 classic Colourchecker patches and a representative subset of the NCS exterior samples in the colour corrected images with their colorimetric readings under a D65 illuminant (consistent with the images' defined white point in sRGB).

The $L^{*} a^{*} b^{*}$ values for the corresponding pixels in the captured images were extracted and averaged over each sample to cancel outliers using ImageJ's colour transform plugin [15-17] and region of interest (ROI) tool, and then compared to their colorimetric readings. We compared the colorimetric and averaged pixel values in $L^{*} a^{*} b^{*}$ using an online $\Delta E$ calculator [18].

The deviations between the colour-corrected Colourchecker image and the colorimetric reference values for the 24 classic patches are listed as $\Delta E$ in Table A2. Our average $\Delta E$ of 1.8 compares favourably with error margins cited in the literature using the same calibration target $[19,20]$, even when taking into account that our spectrophotometer has a certified accuracy of $\Delta E<1$ with saturated colours.

In addition to the Colourchecker, we selected 51 colours from the NCS exterior set to verify the image capture with low to moderately saturated samples. We grouped these colours according to their chromaticness (saturation), blackness, and hue (see Table A3). Hues were selected for each of the elementary NCS colours yellow, red, blue, and green (or within 10\% in the absence of a pure elementary), as well as intermediate colours with a $50 \%$ percent distribution. In addition three neutral greys were included. Grouping the colours aids in identifying deviations which are potentially sensitive to particular colour attributes. Figure 4 shows some of these colours selected for verification in ImageJ. 
The grouped deviations in Table A3 reveal no noticeable dependence on hue, but higher deviations are apparent with lower chromaticness (saturation). This may be attributed to the fact that our ICC profile is optimised for the Colourchecker's saturated colours, whereas the NCS exterior set consists of low to moderately saturated colours.

\subsubsection{Colour Extraction from Image Captures}

Having acquired colour-calibrated captures of all 1400 candidate printed glass colours and the 320 target colours of the NCS exterior set, we extract their pixel values in sRGB colour space. To this end, we define regions of interest (ROIs) for each of the $10 \times 10$ colours in a captured image and average the pixels within them. This filters outlying pixels due to noise and the PV's multiwires. Each ROI covers ca. 50\% of a sample colour's area to avoid overlapping its neighbour due to positioning errors. For the NCS exterior set samples, the ROI area was reduced to $30 \%$ to compensate for fluctuating size and alignment, as these samples were individually cropped and mounted by hand, as exemplified in Figure 4 .

We partly automated the extraction process through a MATLAB script which prompts the user to mark the four corners of the sample grid in each image, and derives the positions of each ROI from these. The script accounts for minor alignment issues due to rotation of the sample with individual vertical and horizontal offsets for each ROI. The images are imported as three separate 2-dimensional matrices for each RGB colour channel. For the candidate printed glass samples, the script iterates over the quartet of image captures to extract the four $5 \times 5$ quadrant subregions covered by the sample PV. The averaged RGB values are dumped to a text file for subsequent colour matching using a separate tool. In addition, the script outputs a diagnostic image containing the ROIs and averaged colours to monitor the extraction process if necessary. A quartet of these is shown in Figure 5 for one printed glass panel.
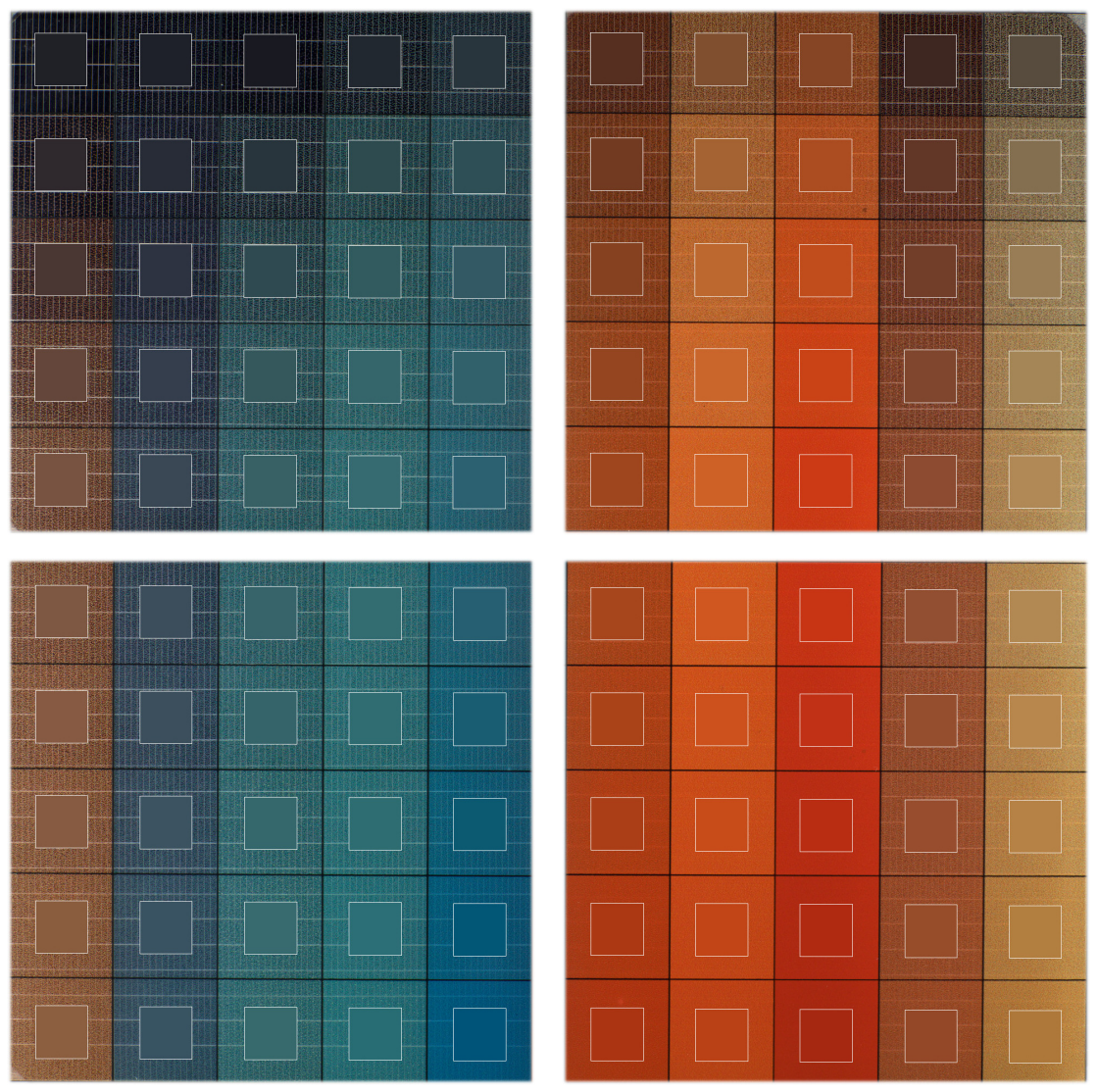

Figure 5. Averaged sample colours extracted from a printed glass panel. Averaging removes noise and reduces the effect of the PV multiwires. Note the user-defined regions of interest superimposed on each sample. These images are contrast enhanced for clarity. 


\subsubsection{Matching by Colour Difference $\Delta E$}

The actual computational matching of candidate printed glass and target NCS exterior set colours was performed by a Python script using the numpy and colormath modules [21,22]. Its operation is summarised in a flowchart with pseudocode in Figure 6.

The script reads the averaged RGB sample values extracted by the MATLAB script from input files and finds best matches by minimising the colour difference $\Delta E$ in the $L^{*} a^{*} b^{*}$ colourspace. This is done by permuting candidate and target colour pairs; for each printed glass colour, we calculate the $\Delta E$ to every NCS exterior set colour. This step is parallelised using Python's multiprocessing.Pool pseudothreads, significantly boosting performance; on our 16-core test system, the $1400 \times 320=448,000$ colour differences were computed in under $17 \mathrm{~s}$.

\section{Read Sample colours, transform to $L^{*} a^{*} b^{*}$ accumulate in lists glassSamples and ncsextSamples}

\section{Build $\Delta \mathrm{E}$ matrix}

\section{Threshold by $\tau$ and filter $\Delta \mathrm{E}$ matrix}

\section{Build best match list bestMatches}
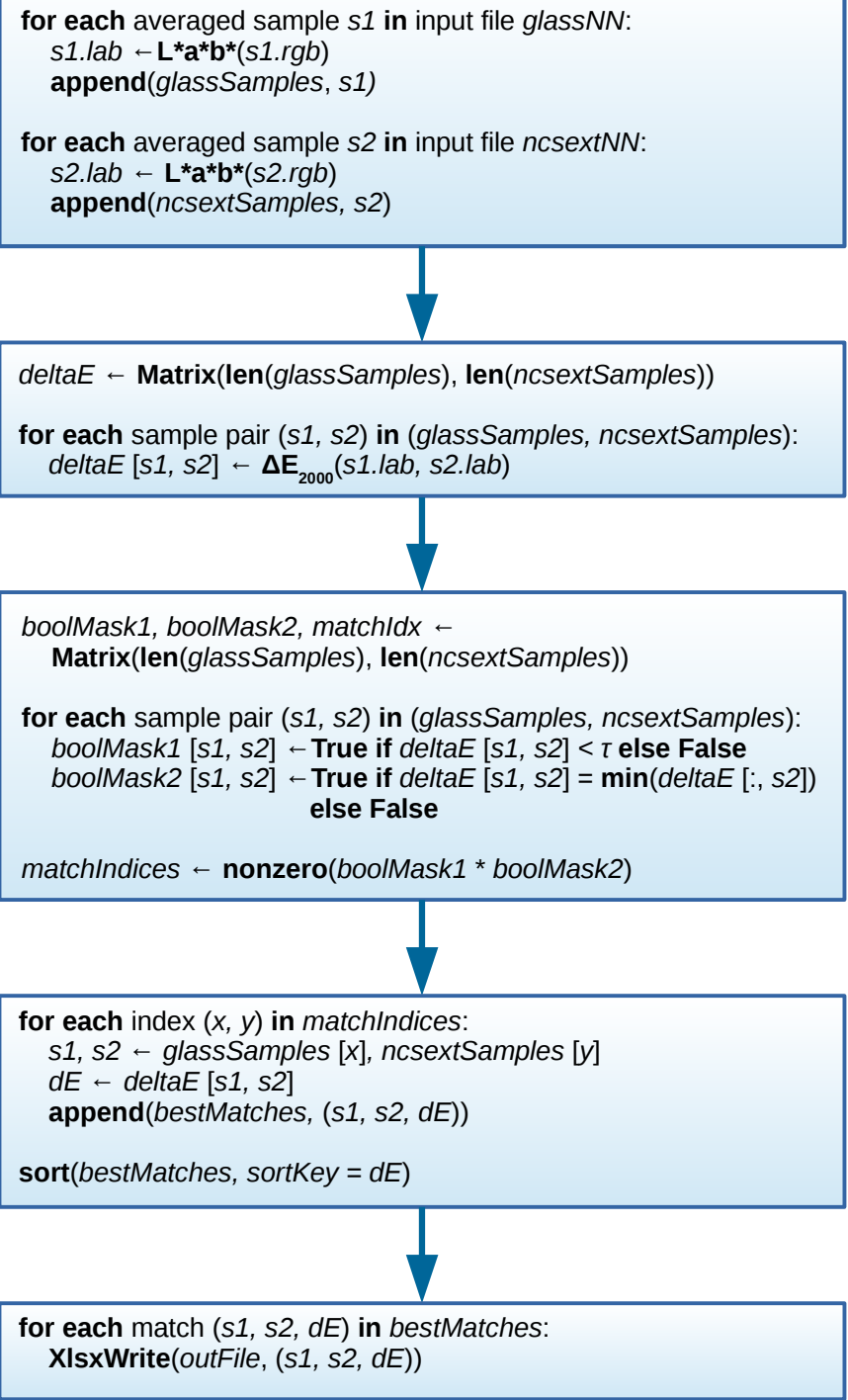

Figure 6. Flowchart of colour matching script based on $\Delta E$. The major tasks (input, $\Delta E$ matrix generation, matrix filtering, best match extraction, and result output) are elaborated in pseudocode. Note that the matrix deltaE is indexed via the column and row corresponding to printed glass sample $s 1$ and NCS exterior sample $s 2$, respectively. The slicing operation deltaE[:, s2] selects all columns for the row corresponding to NCS exterior sample $s 2$. Filtering is accomplished by logically conjuncting the boolean matrices boolMask1 and boolMask2 through multiplication. The function nonzero() returns the indices of all matrix entries which evaluate to True. 
The colour differences are accumulated in a 2-dimensional matrix, with the printed glass samples corresponding to its columns, and the NCS exterior samples corresponding to the rows. Organising the data in this way affords flexibility and caters for future extensions, since it enables symmetric lookups. As such, we can efficiently retrieve all colour differences for a fixed printed glass sample (matrix column) or for a fixed NCS exterior sample (matrix row).

Once all colour differences have been evaluated, the matrix is thresholded by selecting only those entries below a user-specified colour difference threshold $\tau$. These entries are then filtered for the per-row minima, removing multiple matches to the same NCS exterior sample and thus yielding the single best match. Removing duplicates is essential to eliminate redundant results, as their number grows quadratically with $\tau$. The filtered matrix entries are finally sorted according to $\Delta E$ and output as an XLS table using the XlsxWriter Python module [23], which includes colour representations of the matched samples.

\subsection{Visual Matching}

We followed up the computational matching with an experiment using volunteer human observers to visually validate the results. The primary purpose of this experiment was to assess the limits within which untrained observers (our target group) can visually match the printed glass and NCS exterior colours; this dictates the tolerance for our application.

The experiment's secondary purpose was to assess the deviations between the visual matches (expressed as a weighted average) and the computed matches; these should not exceed the tolerance of our application for a computational match to be considered reliable in predicting the perceived combined colour of the printed glass and PV.

\subsubsection{Volunteer Observers}

The 33 participants for this experiment were selected from a pool of volunteers at Hochschule Luzern from various disciplines, with the majority having a background in engineering. The average age of the participants was 31 years (standard deviation $\sigma=10.9$ years), with ages ranging from 15 to 56 years. The male/female ratio of 0.74 was intentionally biased with the benefit that women are better able to distinguish colour gradations, particularly in the middle of the visual spectrum [24].

\subsubsection{Experimental Setup and Materials}

Sixteen printed glass colours from the matches computed with a colour difference threshold of $\tau=3.5$ were selected for the experiment. These were equally divided into two groups consisting of 8 red-brown and 8 green-blue hues. The corresponding printed glass panels (bearing up to 3 matched colours each) were laid out on tables and illuminated under the same conditions as those during the image capture; the same LED studio lights were again arranged for symmetric lighting and set to a CCT of $6500 \mathrm{~K}$, with an average illuminance of 240 lux on the printed glass surfaces. A black surround was suspended over the tables to absorb ceiling reflections. See Figure 7 for an overview of the experimental setup.

To minimise distraction and fatigue for the participants, each printed glass was covered with a dark grey mask with cutouts revealing only those colours under consideration. The colours in each group were individually labelled alphabetically A-H and I-P for easy identification by the participants. Due to an insufficient quantity of available PV modules identical to those used in the image captures, the printed glass colours were placed over dark grey carton to approximate their appearance when combined with such PV modules. The visual difference to the actual PV module used for the computational matching was negligible. 

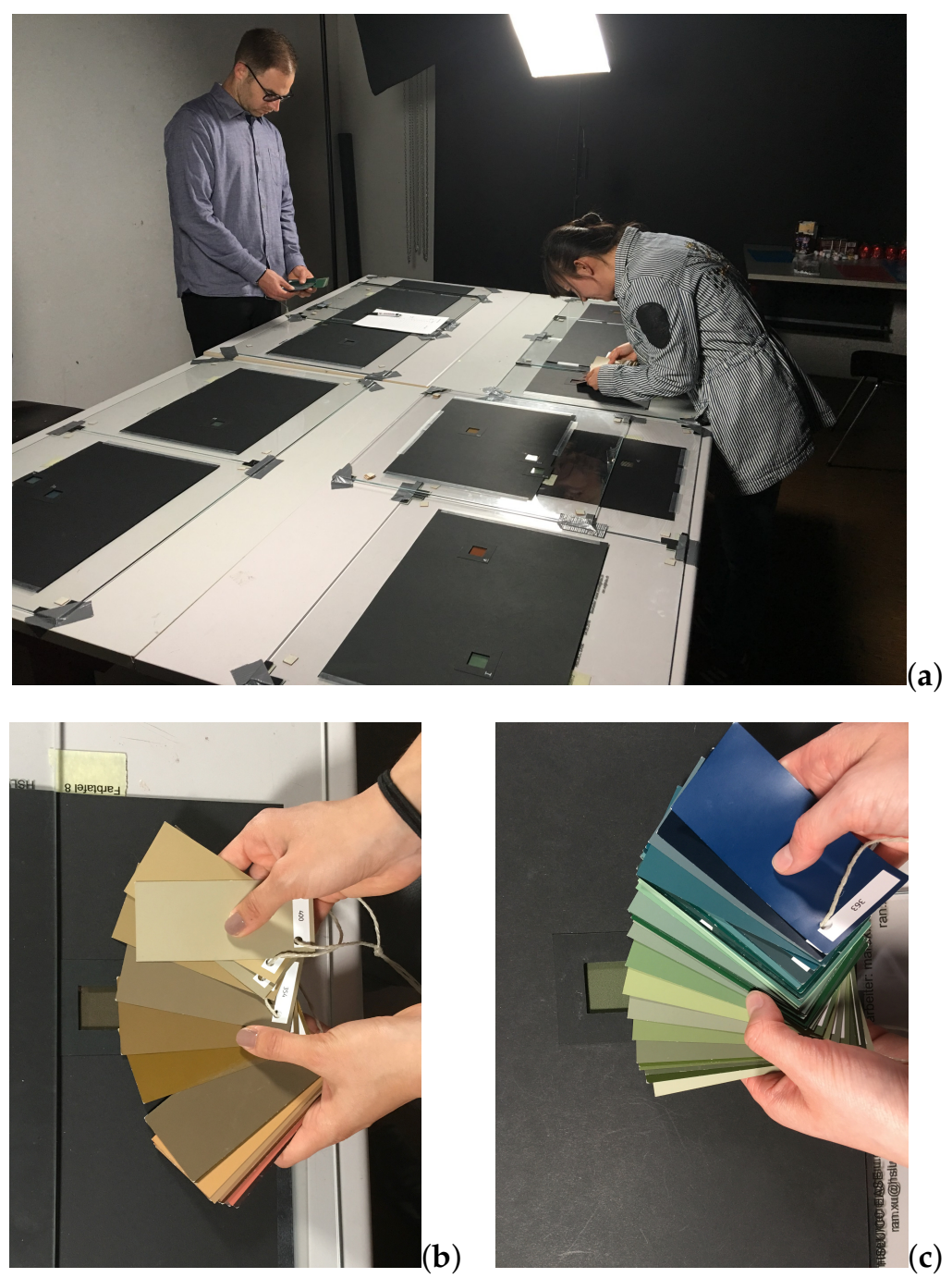

Figure 7. Setup for our visual matching experiment to verify the computed matches (a). The lighting conditions were identical to those for the image capture shown in Figure 3. Volunteer observers were asked to match each of 16 printed glass colours to one or two NCS exterior set colour cards. The colours were equally divided into red-brown (b) and green-blue (c) hues. The appearance of PV modules behind the glass panels was approximated with grey cardboard.

Participants were given two sets of cards from the NCS exterior set, consisting of 44 red-brown and 40 green-blue colours. These included the 16 computed matches, and another 72 selected according to visual similarity. Participants were oblivious to which colours were computationally matched, as this would have very likely led to biased results. The cards were arbitrarily numbered and labeled for easy identification, and bundled with string so that spontaneous matches could be separated and juxtaposed with the printed glass for more thorough examination. With the possible exception of four architects and a designer, the participants were not familiar with the NCS notation printed on the back of the colour cards, nor was it explained to them to prevent biasing their judgement in favour of notation rather than appearance.

\subsubsection{Experimental Procedure}

Once prepared, participants were asked to associate each printed glass colour (by letter) with the visually closest colour card (by number), but were allowed to select two if in doubt, stressing the fact that there is no "right" answer. The participants were cautioned to avoid reflections in the glass and the semi-glossy cards, and maintain a roughly $45^{\circ}$ viewing angle whenever possible. Unlike the 
software which yielded the computed matches, participants were not required to match the printed glass colours to unique colour cards, nor could this be realistically expected. As such, an NCS exterior set card could be visually matched to multiple printed glass colours.

Each participant was given $45 \mathrm{~min}$ for the entire experiment, with $20 \mathrm{~min}$ allotted to each group of colours. The experiment was intermitted by a mandatory $5 \mathrm{~min}$ break between colour groups to avoid potential adaptation effects and symptoms of fatigue. Two participants could perform the experiment in parallel, switching colours halfway.

\section{Results and Discussion}

\subsection{Computational Matching}

Initial results from our computational matching tool were first obtained with a large colour difference threshold $\tau$ to assess the distribution of matched colours as a function of $\Delta E$ within a large set. This selection was then narrowed down to a subset comprised of the best matches which would be indistinguishable (and therefore tolerable) by untrained observers.

\subsubsection{Exhaustive Matches $(\tau=10)$}

Table 1 lists the computationally matched printed glass and NCS exterior set samples as determined by our colour matching script for a colour difference threshold of $\tau=10$. This is an exhaustive table to visually demonstrate the divergence of computed matches as quantified by $\Delta E$. Note that without filtering the $\Delta E$ matrix for duplicate matches, this table would have contained over 9600 redundant matches instead of 74 unique ones, with some NCS exterior colours duplicated over 400 times.

The samples in the table are identified by their panel IDs (glass08-21, ncsext29-32 for the printed glass and NCS exterior set samples, respectively) and by their coordinates $(x, y)$ within the panel. For the printed glass samples, the column $x$ corresponds to a distinct colour, while the row $y$ corresponds to its print density in percent divided by 10 . The glass panels glass $08-14$ and glass $15-21$ are printed with ink volumes of 10 and $20 \mathrm{pl}$, respectively.

According to experimental data, a colour difference of $\Delta E_{1976}>3.5$ is just noticeable by a casual observer, while a $\Delta E_{1976}>5$ would be perceived as two different colours [25]. Since these tolerances were determined for the obsolete CIE 1976 colour difference standard, and $\Delta E_{2000}$ is generally lower than $\Delta E_{1976}$ for the same pairs of colours, we expect a $\Delta E_{2000}$ above 5 to be obvious to any observer.

While it may not be evident from the colour representations in the table depending on the medium (particularly in print), there are noticeable deviations in the lower rows of the table, notably for $\Delta E>7$. This range is dominated by the more saturated samples from the NCS exterior set, which are (poorly) matched to more muted printed glass samples. This is expected, since $\Delta E_{2000}$ takes differences in perceived saturation into account, unlike earlier formulations of colour difference.

Table 1 is graphically summarised as histogram in Figure A1. This plots the distribution of $\Delta E$ as a function of print density and ink volume. While the graphs for both print densities vaguely resemble a normal distribution of deviations, the dataset is too small to be conclusive. As such there is no discernible correlation between $\Delta E$, print density, and ink volume. With both ink volumes, most matches were found for moderate print densities, namely $50-60 \%$ with $10 \mathrm{pl}$, and $60-70 \%$ with $20 \mathrm{pl}$. As a tentative indicator of PV efficiency, we note that eight close matches with a $\Delta E \leq 4$ were found for print densities below $50 \%$. However, this needs to be confirmed with electrical measurements in future work. 
Table 1. Computed matches for a colour difference threshold of $\tau=10$ as output by our matching script. The actual printed glass colours for the top 20 matches $(\Delta E<3.5)$ are shown in Table 2.

\begin{tabular}{|c|c|c|c|c|}
\hline \multicolumn{5}{|c|}{ Glass Sample } \\
\hline ID & $\mathbf{L}$ & $*^{*} \mathbf{a}$ & $*_{\mathbf{b}}$ & Colour \\
\hline glass15 $(01,06)$ & 41.80 & 3.53 & 17.26 & \\
\hline glass09 $(01,09)$ & 27.83 & 5.49 & 9.34 & \\
\hline glass $12(10,03)$ & 34.46 & 18.23 & 23.53 & \\
\hline glass $10(08,06)$ & 41.66 & -8.27 & 15.02 & \\
\hline glass $11(10,04)$ & 35.26 & -9.66 & 2.50 & \\
\hline glass $19(04,09)$ & 37.97 & 11.08 & 29.66 & \\
\hline glass15 $(09,04)$ & 30.06 & 10.18 & 15.47 & \\
\hline glass $18(07,04)$ & 24.65 & -7.03 & -2.31 & \\
\hline glass20 $(02,06)$ & 27.76 & 14.35 & 13.77 & \\
\hline glass $19(02,10)$ & 36.86 & -14.43 & 4.38 & \\
\hline glass08 $(01,06)$ & 37.97 & 1.29 & 11.18 & \\
\hline glass $14(10,03)$ & 42.21 & 6.98 & 20.82 & \\
\hline glass $15(05,10)$ & 32.44 & 9.89 & 20.12 & \\
\hline glass $16(05,07)$ & 26.51 & -9.42 & -11.31 & \\
\hline glass $18(02,10)$ & 34.72 & -3.75 & 6.34 & \\
\hline glass $13(02,03)$ & 22.44 & 8.10 & 6.91 & \\
\hline glass19 $(07,03)$ & 40.53 & 4.23 & 23.72 & \\
\hline glass $15(03,10)$ & 31.75 & 4.87 & 16.15 & \\
\hline glass $16(04,10)$ & 30.27 & -12.54 & -6.54 & \\
\hline glass $12(01,06)$ & 46.56 & -15.88 & 6.66 & \\
\hline glass $15(01,03)$ & 34.42 & 2.75 & 12.20 & \\
\hline glass $16(02,04)$ & 20.82 & -0.11 & -5.80 & \\
\hline glass $17(01,03)$ & 33.46 & 16.66 & 19.72 & \\
\hline glass $14(10,05)$ & 44.09 & 12.17 & 26.08 & \\
\hline glass $17(08,02)$ & 33.02 & -5.86 & 11.02 & \\
\hline glass09 $(05,05)$ & 27.80 & -5.32 & -6.50 & \\
\hline glass $17(08,03)$ & 39.47 & -8.52 & 14.92 & \\
\hline glass $18(08,07)$ & 25.94 & -1.43 & 0.77 & \\
\hline glass $10(01,05)$ & 35.14 & 14.17 & 17.60 & \\
\hline glass $17(05,06)$ & 34.26 & -13.11 & 2.24 & \\
\hline glass $12(01,02)$ & 34.01 & -9.56 & 1.94 & \\
\hline glass $12(01,04)$ & 41.35 & -12.87 & 4.54 & \\
\hline glass16 $(04,09)$ & 30.47 & -12.23 & -6.66 & \\
\hline glass08 $(09,07)$ & 31.27 & 7.84 & 12.98 & \\
\hline glass $10(01,05)$ & 35.14 & 14.17 & 17.60 & \\
\hline glass $20(02,04)$ & 26.76 & 12.27 & 11.49 & \\
\hline glass $16(01,05)$ & 28.22 & 7.30 & 9.59 & \\
\hline glass $15(01,06)$ & 41.80 & 3.53 & 17.26 & \\
\hline glass $14(10,05)$ & 44.09 & 12.17 & 26.08 & \\
\hline glass $15(01,06)$ & 41.80 & 3.53 & 17.26 & \\
\hline glass12(01,06) & 46.56 & -15.88 & 6.66 & \\
\hline glass $19(02,05)$ & 34.84 & -12.30 & 1.90 & \\
\hline glass $17(05,10)$ & 36.70 & -16.54 & 4.31 & \\
\hline glass $17(08,06)$ & 47.77 & -11.64 & 21.99 & \\
\hline glass $10(08,06)$ & 41.66 & -8.27 & 15.02 & \\
\hline glass $16(04,10)$ & 30.27 & -12.54 & -6.54 & \\
\hline glass $16(04,09)$ & 30.47 & -12.23 & -6.66 & \\
\hline glass $16(02,03)$ & 18.14 & 0.58 & -5.24 & \\
\hline glass17(09,09) & 54.85 & 3.22 & 49.26 & \\
\hline glass $16(02,06)$ & 24.01 & -1.75 & -6.46 & \\
\hline glass11 $(10,06)$ & 38.60 & -11.04 & 3.71 & \\
\hline glass $13(01,05)$ & 41.74 & 22.84 & 31.03 & \\
\hline glass $20(02,07)$ & 27.98 & 15.32 & 14.87 & \\
\hline glass $18(02,06)$ & 32.14 & -3.83 & 2.72 & \\
\hline glass10 $(01,03)$ & 31.40 & 11.25 & 13.53 & \\
\hline glass $13(01,04)$ & 41.34 & 18.83 & 28.11 & \\
\hline glass10 $(01,03)$ & 31.40 & 11.25 & 13.53 & \\
\hline glass $12(01,07)$ & 47.86 & -17.83 & 7.98 & \\
\hline glass13(02,05) & 26.19 & 10.06 & 9.13 & \\
\hline glass $17(06,07)$ & 50.04 & 0.67 & 32.59 & \\
\hline glass $15(01,05)$ & 40.22 & 3.49 & 15.69 & \\
\hline glass $17(10,06)$ & 44.78 & 11.75 & 31.28 & \\
\hline glass $16(02,04)$ & 20.82 & -0.11 & -5.80 & \\
\hline glass $10(10,06)$ & 44.66 & 5.69 & 23.20 & \\
\hline glass $13(01,05)$ & 41.74 & 22.84 & 31.03 & \\
\hline glass10 $(01,06)$ & 35.40 & 15.76 & 19.20 & \\
\hline glass $18(02,08)$ & 34.03 & -3.56 & 4.77 & \\
\hline glass $14(10,05)$ & 44.09 & 12.17 & 26.08 & \\
\hline glass $14(10,05)$ & 44.09 & 12.17 & 26.08 & \\
\hline glass09 $(05,10)$ & 23.07 & -6.16 & -12.53 & \\
\hline glass20(02,07) & 27.98 & 15.32 & 14.87 & \\
\hline glass $19(01,06)$ & 50.17 & -23.17 & 12.25 & \\
\hline glass $17(09,08)$ & 55.37 & 1.70 & 46.10 & \\
\hline glass $20(02,03)$ & 24.56 & 11.10 & 10.06 & \\
\hline
\end{tabular}

\begin{tabular}{|c|c|c|c|c|c|}
\hline \multicolumn{5}{|c|}{ NCSext Sample } & \multirow{2}{*}{ DeltaE } \\
\hline Colour & ID & $\mathrm{L}$ & $* a$ & $* \mathbf{b}$ & \\
\hline & ncsext31 $(04,08)$ & 41.84 & 3.79 & 17.78 & 0.37 \\
\hline & ncsext32 $(02,02)$ & 26.99 & 5.73 & 9.49 & 0.69 \\
\hline & ncsext32 $(02,10)$ & 34.59 & 17.26 & 23.96 & 0.85 \\
\hline & ncsext31 $(06,03)$ & 42.24 & -9.14 & 14.86 & 0.98 \\
\hline & ncsext31 $(07,04)$ & 35.39 & -8.53 & 2.01 & 1.09 \\
\hline & ncsext31 $(06,01)$ & 37.31 & 10.31 & 27.46 & 1.13 \\
\hline & ncsext31 $(05,05)$ & 30.05 & 11.61 & 17.18 & 1.29 \\
\hline & ncsext32 $(01,03)$ & 25.05 & -8.41 & -2.69 & 1.39 \\
\hline & ncsext31 $(07,09)$ & 26.58 & 14.93 & 12.55 & 1.44 \\
\hline & ncsext31 $(08,09)$ & 37.66 & -15.77 & 5.98 & 1.53 \\
\hline & ncsext31 $(04,07)$ & 37.75 & 2.49 & 10.03 & 1.89 \\
\hline & ncsext31 $(04,03)$ & 42.10 & 8.34 & 19.16 & 1.92 \\
\hline & ncsext31 $(04,04)$ & 31.53 & 8.15 & 20.97 & 2.00 \\
\hline & ncsext31 $(05,03)$ & 25.84 & -10.99 & -9.55 & 2.12 \\
\hline & ncsext31 $(06,02)$ & 36.85 & -5.22 & 7.52 & 2.50 \\
\hline & ncsext32 $(02,03)$ & 21.84 & 9.71 & 4.34 & 2.88 \\
\hline & ncsext31 $(05,06)$ & 38.44 & 4.18 & 28.43 & 2.88 \\
\hline & ncsext31 $(05,07)$ & 31.19 & 3.87 & 20.65 & 3.09 \\
\hline & ncsext32 $(01,06)$ & 29.31 & -15.11 & -3.90 & 3.16 \\
\hline & ncsext29(06,09) & 49.14 & -13.67 & 4.07 & 3.39 \\
\hline & ncsext31 $(08,06)$ & 36.86 & 4.20 & 9.60 & 3.53 \\
\hline & ncsext32 $(02,05)$ & 21.11 & -0.03 & -10.71 & 3.58 \\
\hline & ncsext32 $(01,08)$ & 33.99 & 19.60 & 16.21 & 3.77 \\
\hline & ncsext30 $(08,08)$ & 46.11 & 12.73 & 20.55 & 3.86 \\
\hline & ncsext31 $(07,08)$ & 30.09 & -8.67 & 14.62 & 3.88 \\
\hline & ncsext31 $(04,10)$ & 32.71 & -4.87 & -5.33 & 3.94 \\
\hline & ncsext31 $(07,02)$ & 37.03 & -11.67 & 20.26 & 3.98 \\
\hline & ncsext32 $(01,10)$ & 28.61 & 0.80 & -0.69 & 4.02 \\
\hline & $\operatorname{ncsext} 31(10,10)$ & 39.29 & 11.19 & 14.15 & 4.34 \\
\hline & ncsext32 $(01,01)$ & 30.96 & -15.86 & 6.72 & 4.44 \\
\hline & ncsext32 $(01,02)$ & 36.28 & -8.45 & -2.99 & 4.67 \\
\hline & ncsext31 $(06,04)$ & 43.14 & -8.30 & 1.58 & 4.67 \\
\hline & ncsext31 $(05,02)$ & 31.79 & -16.12 & -13.26 & 4.76 \\
\hline & ncsext32 $(02,08)$ & 36.47 & 7.09 & 9.70 & 4.77 \\
\hline & ncsext31 $(07,07)$ & 38.81 & 14.82 & 12.59 & 4.83 \\
\hline & ncsext32 $(02,06)$ & 27.33 & 15.64 & 7.04 & 4.87 \\
\hline & ncsext31 $(10,09)$ & 33.18 & 7.90 & 5.46 & 5.23 \\
\hline & ncsext31 $(04,06)$ & 45.86 & 2.37 & 11.12 & 5.37 \\
\hline & ncsext30 $(04,04)$ & 49.47 & 10.86 & 22.43 & 5.53 \\
\hline & ncsext31 $(08,07)$ & 45.25 & 4.47 & 10.54 & 5.75 \\
\hline & ncsext30 $(06,07)$ & 49.27 & -14.86 & -0.39 & 5.80 \\
\hline & ncsext31 $(07,06)$ & 38.65 & -14.43 & -4.02 & 5.81 \\
\hline & ncsext32 $(01,09)$ & 33.17 & -24.25 & 9.39 & 5.89 \\
\hline & ncsext29(02,10) & 51.49 & -8.26 & 13.68 & 6.05 \\
\hline & ncsext31 $(07,01)$ & 45.13 & -5.16 & 7.72 & 6.08 \\
\hline & ncsext31 $(07,05)$ & 30.56 & -21.19 & -4.52 & 6.38 \\
\hline & ncsext31 $(05,01)$ & 37.75 & -10.54 & -9.06 & 6.44 \\
\hline & ncsext32 $(02,04)$ & 20.47 & 4.28 & -11.95 & 6.49 \\
\hline & ncsext29(04,04) & 56.23 & 8.85 & 36.76 & 6.76 \\
\hline & ncsext31 $(06,08)$ & 31.89 & 0.49 & -9.43 & 7.03 \\
\hline & ncsext31 $(07,03)$ & 43.13 & -8.00 & -2.82 & 7.24 \\
\hline & ncsext30 $(07,09)$ & 48.00 & 22.43 & 23.27 & 7.27 \\
\hline & ncsext32 $(01,07)$ & 31.93 & 22.59 & 10.45 & 7.32 \\
\hline & ncsext31 $(04,02)$ & 35.91 & 0.38 & -1.33 & 7.35 \\
\hline & ncsext32 $(02,07)$ & 34.90 & 10.08 & 4.08 & 7.49 \\
\hline & ncsext $29(08,08)$ & 45.00 & 17.76 & 16.31 & 7.58 \\
\hline & ncsext31 $(07,10)$ & 35.07 & 15.42 & 6.63 & 7.63 \\
\hline & ncsext29 $(02,07)$ & 53.73 & -12.16 & 11.06 & 7.71 \\
\hline & ncsext31 $(05,04)$ & 31.30 & 12.76 & 1.42 & 7.83 \\
\hline & ncsext30 $(04,05)$ & 53.13 & 2.91 & 18.19 & 7.89 \\
\hline & ncsext31 $(04,05)$ & 44.34 & 6.52 & 8.55 & 7.91 \\
\hline & ncsext29 $(09,10)$ & 52.51 & 13.21 & 28.62 & 7.94 \\
\hline & ncsext31 $(06,10)$ & 20.85 & 0.90 & -18.07 & 8.00 \\
\hline & ncsext30 $(04,03)$ & 51.77 & 7.60 & 17.97 & 8.09 \\
\hline & ncsext29(02,06) & 42.54 & 24.30 & 17.72 & 8.43 \\
\hline & ncsext29 $(09,05)$ & 37.75 & 24.27 & 12.88 & 8.59 \\
\hline & ncsext31 $(05,10)$ & 38.19 & 0.93 & -1.26 & 8.68 \\
\hline & ncsext30 $(08,07)$ & 50.93 & 9.66 & 15.39 & 8.80 \\
\hline & ncsext30 $(09,10)$ & 51.51 & 15.76 & 19.54 & 9.27 \\
\hline & ncsext31 $(06,09)$ & 26.13 & 1.33 & -23.48 & 9.46 \\
\hline & ncsext29 $(07,06)$ & 34.36 & 25.73 & 10.82 & 9.46 \\
\hline & ncsext30 $(06,05)$ & 58.56 & -15.48 & 6.35 & 9.62 \\
\hline & ncsext29(05,10) & 62.55 & 9.53 & 36.64 & 9.69 \\
\hline & ncsext32 $(01,05)$ & 22.31 & 21.26 & 2.82 & 9.96 \\
\hline
\end{tabular}


Table 2. 20 computed matches for a colour difference tolerance $\tau=3.5$, along with the printer settings for the glass samples and the corresponding NCS exterior set codes. These matches correspond to the upper 20 rows of Table 1, but the printed glass samples are shown here as the actual printed colours without PV.

\begin{tabular}{|c|c|c|c|c|c|c|}
\hline \multicolumn{7}{|c|}{ Glass Sample Print Settings } \\
\hline ID & R & G & B & $\begin{array}{c}\text { Density } \\
\text { [\%] }\end{array}$ & $\begin{array}{c}\text { Volume } \\
\text { [pL] }\end{array}$ & $\begin{array}{c}\text { Printed } \\
\text { Colour }\end{array}$ \\
\hline glass15(01,06) & 233 & 211 & 181 & 60 & 20 & \\
glass09(01,09) & 253 & 233 & 224 & 90 & 10 & \\
glass12(10,03) & 233 & 101 & 65 & 30 & 10 & \\
glass10(08,06) & 242 & 238 & 179 & 60 & 10 & \\
glass11(10,04) & 219 & 237 & 189 & 40 & 10 & \\
glass19(04,09) & 223 & 187 & 131 & 90 & 20 & \\
glass15(09,04) & 231 & 197 & 173 & 40 & 20 & \\
glass18(07,04) & 230 & 241 & 231 & 40 & 20 & \\
glass20(02,06) & 243 & 205 & 215 & 60 & 20 & \\
glass19(02,10) & 223 & 240 & 223 & 100 & 20 & \\
glass08(01,06) & 233 & 211 & 181 & 60 & 10 & \\
glass14(10,03) & 209 & 142 & 51 & 30 & 10 & \\
glass15(05,10) & 236 & 208 & 185 & 100 & 20 & \\
glass16(05,07) & 125 & 188 & 240 & 70 & 20 & \\
glass18(02,10) & 227 & 224 & 213 & 100 & 20 & \\
glass13(02,03) & 243 & 205 & 215 & 30 & 10 & \\
glass19(07,03) & 234 & 190 & 103 & 30 & 20 & \\
glass15(03,10) & 253 & 239 & 222 & 100 & 20 & \\
glass16(04,10) & 201 & 234 & 239 & 100 & 20 & \\
glass12(01,06) & 200 & 252 & 175 & 60 & 10 & \\
\hline
\end{tabular}

\begin{tabular}{|c|c|c|}
\hline \multicolumn{2}{|c|}{ Matched NCS Ext. Sample } & \multirow{2}{*}{$\Delta E_{2000}$} \\
\hline ID & NCS Code & \\
\hline ncsext31(04,08) & S 6020-Y10R & 0.37 \\
\hline ncsext32 $(02,02)$ & S 8010-Y50R & 0.69 \\
\hline$n \operatorname{csext} 32(02,10)$ & S 6030-Y50R & 0.85 \\
\hline ncsext31 $(06,03)$ & S 6020-G50Y & 0.98 \\
\hline ncsext31 $(07,04)$ & S 7010-G10Y & 1.09 \\
\hline ncsext31 $(06,01)$ & S 6030-Y30R & 1.13 \\
\hline ncsext31 $(05,05)$ & S 7020-Y50R & 1.29 \\
\hline ncsext32 $(01,03)$ & S 8010-B70G & 1.39 \\
\hline ncsext31 $(07,09)$ & S 7020-Y70R & 1.44 \\
\hline ncsext31 $(08,09)$ & S 6020-G10Y & 1.53 \\
\hline ncsext31 $(04,07)$ & S 7010-Y10R & 1.89 \\
\hline ncsext31 $(04,03)$ & S 6020-Y30R & 1.92 \\
\hline ncsext31 $(04,04)$ & S 7020-Y30R & 2.00 \\
\hline ncsext31 $(05,03)$ & S 7020-B30G & 2.12 \\
\hline ncsext31 $(06,02)$ & S 7010-G50Y & 2.50 \\
\hline ncsext32 $(02,03)$ & S 8010-Y90R & 2.88 \\
\hline ncsext31 $(05,06)$ & S 6030-Y10R & 2.88 \\
\hline ncsext31 $(05,07)$ & S 7020-Y10R & 3.09 \\
\hline ncsext32 $(01,06)$ & S 7020-B70G & 3.16 \\
\hline ncsext29 $(06,09)$ & S 5020-G10Y & 3.39 \\
\hline
\end{tabular}

\subsubsection{Best Matches $(\tau=3.5)$}

Based on the published colour difference tolerances [25], we refined our exhaustive matches by reducing the colour difference threshold to $\tau=3.5$, since such a $\Delta E$ would just be noticeable to an untrained observer, and thus consistent with our application's intent. This narrowed Table 1 down to just the top 20 best matches for further analysis.

The relatively low number of matches for a tight colour difference tolerance arises from a suboptimal correspondence of candidate and target colours; the selections of colours for both sample sets were driven by different motivations. Similarly, our choice of colours was not motivated by the objective of maximising exact matches, but rather finding any number of closest matches from the set of available colours. Unsurprisingly, given the selection of colours that comprise the NCS exterior set, the matches are predominantly of earthen hues: red-yellow, with some muted shades of green-blue.

Table 2 lists these best matches translated to the print settings for the glass samples (RGB colour, print density, and ink volume), and the matched NCS exterior set colour code. The table also includes a representation of the actually printed colour without the PV module. Note that the glass manufacturer converted these colours to the printer's native subtractive CMYK colourspace, which may deviate depending on the printer's calibration. Hence the RGB print settings are not predictive of the combined appearance with PV and cannot be factored into the computational matching.

The colour differences are equidistributed with regard to ink volume. The print density averages $56.5 \%$, while the minimum is $30 \%$ in four instances. As expected, the table reveals an inverse relationship between the saturation of the printed glass sample and the blackness attribute of the matched NCS exterior sample; the less saturated the glass sample, the higher the influence of the PV sample on the resultant blended colour, which translates to a higher NCS blackness attribute. It is also apparent that the most saturated printed glass samples tend to match when printed with a low density.

\subsection{Visual Matching}

The results of the visual matching experiment were compiled from evaluation sheets each participant used to record the matched glass samples and NCS exterior set colour cards. Five visual 
matches from different participants were discarded as their labels were not in the participants' assigned colour group, presumably because the numbers were noted in error. Three of these participants specified a valid alternate match.

In general, participants found the experiment more demanding than it initially appeared to them, primarily due the subtleties of the colours under consideration. They experienced particular difficulty matching the dark, subdued colours included in the NCS exterior set. In only six cases (four red-brown, two green-blue) did the majority of observers agree with the computed matches. The dominant visual matches did, however, in almost all cases agree with the computed match in terms of NCS hue. This indicates the colour correction during the image acquisition for the computed matches was of sufficient accuracy for our application.

\subsubsection{Statistical Evaluation}

Tables A4 and A5 summarise the statistical analysis of our visual matching experiment for the red-brown and green-blue colour groups, respectively. For each printed glass sample, a visual average colour $\mu_{v}$ was approximated from the set of visually matched colour cards $M_{v}$ weighted by their frequency:

$$
\mu_{v}\left(M_{v}\right)=\sum_{m_{i} \in M_{v}} w_{i} \cdot L^{*} a^{*} b^{*}\left(m_{i}\right),
$$

where $m_{i} \in M_{v}$ is a visually matched NCS exterior set colour from the set of visual matches $M_{v}$ for a printed glass sample, and $w_{i}$ is its normalised weight based on how often it was selected as a match. Note that the $L^{*} a^{*} b^{*}$ colour space is particularly suitable for interpolation as it is defined to be perceptually uniform. The $L^{*} a^{*} b^{*}$ values for each $m_{i}$ were obtained from colorimetric readings from the actual matched NCS exterior set colour cards used in the experiment. This accounts for tolerances in printing the cards, as well as deterioration after being handled during the experiment.

A modified weighted population standard deviation $\sigma_{v}$ was then computed based on each matched NCS exterior colour's colour difference $\Delta E$ to $\mu_{v}$ :

$$
\sigma_{v}\left(M_{v}\right)=\sqrt{\sum_{m_{i} \in M_{v}} w_{i} \cdot \Delta E^{2}\left(m_{i}, \mu_{v}\left(M_{v}\right)\right)},
$$

The standard deviation $\sigma_{v}$ defines the tolerance of our visual matches, under the assumption that the participants were-with possible exception of those few familiar with NCS-inexperienced with the task at hand. The computed tolerance varies from 4 to 6 , which agrees with the typical $\Delta E$ threshold of 5 published in the literature for an obvious difference in colour as perceived by casual observers [25].

\subsubsection{Visual vs. Computed Matches}

Tables A4 and A5 also highlight the computated matches from our image based method in red; for each printed glass sample, the tables include the computationally matched NCS exterior set sample (cf. Table 2), the number of times it was visually matched, and its colour difference $\Delta E$ to the visual average $\mu_{v}$. Ideally, the latter deviations should in all cases be within the tolerance defined by $\sigma_{v}$. These deviations average to a $\Delta E$ of 5.12 for all 16 printed glass samples, which again correlates with the published tolerance for an obvious perceived colour difference.

These results are summarised graphically as scatter plots in Figure 8. The points represent the positions of the weighted visual averages $\mu_{v}$ in $L^{*} a^{*} b^{*}$ space, with their colour proportional to the colour difference $\Delta E$ to the corresponding image-based matches $m_{i}$. Green points indicate good agreement between visual and image-based matches, while red ones are indicative of large deviations $(\Delta E>10)$. 


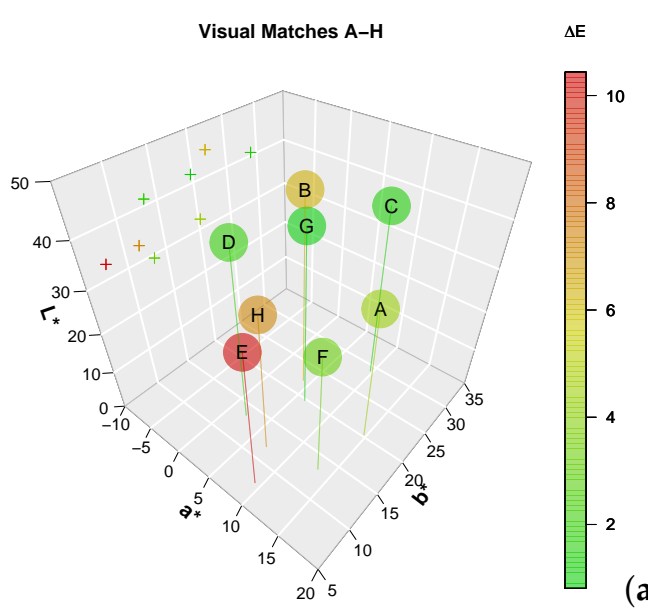

(a)

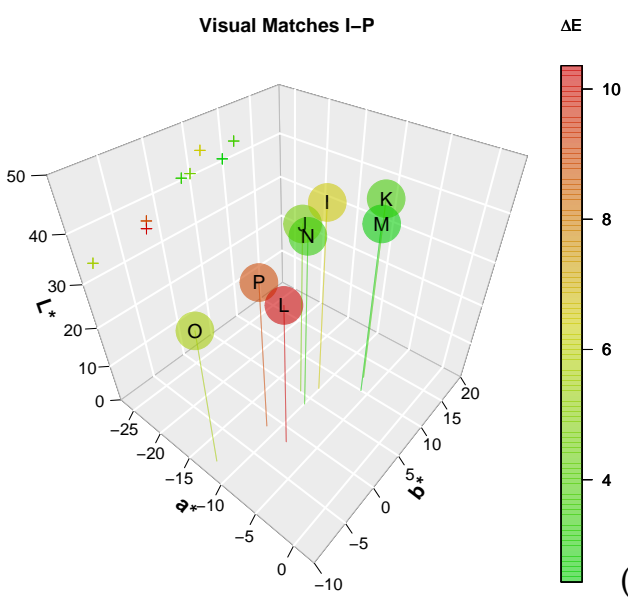

(b)

Figure 8. Visual matching results for red-brown samples A-H (a) and green-blue samples I-P (b). Each point represents the position of the weighted visual averages in $L^{*} a^{*} b^{*}$ space, with its colour indicating the colour difference $\Delta E$ to the corresponding computed matches. The visual averages are also projected onto the $\left(L^{*}, b^{*}\right)$ plane for clarity.

The graphs indicate that deviations are generally larger for visual averages with lower luminance $\left(L^{*}<40\right)$ and low saturation $\left(\left|a^{*}\right|,\left|b^{*}\right|<10\right)$. This correlates with the difficulties that observers had in distinguishing these particular colours, but is also indicative of a fundamentally poor correspondence between these individual printed glass samples and the NCS exterior set.

\section{Conclusions and Future Work}

We have presented a method to match the appearance of coloured PV frontglass to a set of given exterior colours with the aim of integrating the former into building façades as BIPV. Our computational image-based approach compares a large number of unknown candidate printed glass samples and known target colours using the CIE $\Delta E$ colour difference metric. The disparities inherent in the candidate and target sample sets constrain the number of expected matches; consequently, we aim to find any number of closest matches, rather than a maximum of exact matches.

We have demonstrated our method with 1400 candidates and 320 targets from the Farbraum Stadt and NCS Exterior Set collections, respectively. This resulted in 20 computed matches with a colour difference $\Delta E<3.5$. This relatively low number of matches is primarily due to the mentioned discrepancies between the printed glass and NCS exterior sample sets, rather than a limitation of our methodology. 
We visually verified the computed matches with human volunteers in an experiment, which revealed an observer tolerance of $\Delta E \approx 5$, and similar average deviations to the computed matches. This agrees with the published limits for an obvious colour difference for casual observers, who are the target group of our application. In four cases, the computed and visual matches deviated significantly by a $\Delta E>7$, which indicate that our image capture process requires optimisation, e.g., by using an IT8.7 colour calibration target, and more accurate lighting.

In general, the results of our computational and visual matching indicate that observers have a significant tolerance for matched BIPV colours, and that this leeway may be leveraged towards lower printing densities and PV losses. Our results reveal that reasonable matches can be found for printed glass samples of low to moderate print densities (30-60\% for 12 out of the top 20 matches) for ink volumes of 10 and $20 \mathrm{pl}$.

These results suggest acceptable losses when combined with PV, which we plan to confirm by electrical efficiency measurements in future work. This would need to be quantified in terms of the PV's spectral response to the printed colour, as well the type of PV used (monocrystalline or polycrystalline). The latter would also require further investigation in terms of matched colours, since the PV type noticeably impacts the perceived colour when combined with printed glass, particularly at low print densities.

The results documented here should be considered exemplary of the predominantly muted target colours that comprise the NCS exterior set. Consequently, the matches generally fall into red-brown or green-blue hues of low to moderate saturation. A more comprehensive application to a wider gamut of colours is planned, along with a fully automated extraction of averaged pixel colours from captured images.

Acknowledgments: This research was supported by the Swiss National Science Foundation as part of the project ACTIVE INTERFACES - Holistic strategy to simplify standards, assessments and certifications for building integrated photovoltaics (\#153849). We would like to thank the following for their contributions to this publication: Glas Trösch AG, Bützberg, Switzerland for providing the printed glass samples; our colleague Markus Käch for photographing the printed glass and NCS exterior set samples in his studio as part of our image capture process; our colleague Ran Xu for developing the MATLAB script to extract the averaged RGB values from the captured images; and the volunteers who participated in the visual matching experiment, which was co-supervised by Ran.

Author Contributions: Stephen Wittkopf initiated this research and supported it with consultations. Marek Krehel contributed to writing, data analysis and visualisations. Roland Schregle wrote the article, implemented the colour matching script and analysed its results. He also conducted the image captures and co-supervised the visual matching experiment. All authors reviewed the publication.

Conflicts of Interest: The authors declare no conflict of interest. 


\section{Appendix A. Candidate Printed Glass Colours}

Table A1 lists the 70 colours selected for the printed glass samples. 67 of these colours are taken from the Farbraum Stadt set [10] and represent ca. 96\% of exterior façades in the city of Zürich, Switzerland. Their published NCS codes were converted to RGB for printing.

An additional 3 colours were included to round the total to 70 to fully utilise the space on the glass panels. These colours are derived from three colours selected from Farbraum Stadt, with 20\% increased RGB intensities; their NCS equivalents are therefore approximate.

Table A1. Candidate colours selected for printing on glass, along with their corresponding RGB print settings.

\begin{tabular}{|c|c|c|c|c|c|c|c|c|c|c|c|}
\hline NCS & $\mathbf{R}$ & G & $B$ & NCS & $\mathbf{R}$ & G & B & NCS & $\mathbf{R}$ & G & B \\
\hline S 0500-N & 241 & 239 & 235 & S 1010-Y20R & 233 & 215 & 183 & S 3030-G30Y & 128 & 159 & 113 \\
\hline S 0502-B & 232 & 237 & 239 & 1010-Y40R & 234 & 210 & 183 & 3030-R & 182 & 121 & 124 \\
\hline S 0502-G & 233 & 237 & 234 & 1010-Y50R & 236 & 208 & 185 & S 3030-Y20R & 187 & 148 & 94 \\
\hline S 0502-R & 241 & 234 & 232 & S 1020-G40Y & 199 & 215 & 172 & S 3040-Y20R & 190 & 141 & 74 \\
\hline S 0502-Y & 243 & 239 & 227 & S 1040-R20B & 233 & 151 & 175 & S 3040-Y90R & 182 & 100 & 94 \\
\hline S 0502-Y50R & 243 & 237 & 229 & 1060-Y90R & 231 & 105 & 102 & S 3050-Y20R & 189 & 133 & 55 \\
\hline S 0505-G80Y & 240 & 238 & 220 & 1070-Y70R & 233 & 101 & 65 & s 3502-R & 165 & 161 & 161 \\
\hline S 0505-Y50R & 246 & 229 & 212 & S 1510-Y30R ${ }^{1}$ & 225 & 201 & 168 & S 3502-Y & 165 & 163 & 155 \\
\hline S 0510-G10Y & 223 & 240 & 223 & S 2000-N & 201 & 200 & 197 & S 4000-N & 153 & 153 & 152 \\
\hline S 0520-G80Y & 242 & 238 & 179 & S 2002-Y & 198 & 195 & 187 & S 4010-Y50R & 168 & 144 & 127 \\
\hline S 052 & 243 & 205 & 215 & 2005-G20Y & 188 & 197 & 189 & -Y70R & 164 & 138 & 128 \\
\hline S 0520-Y30R & 253 & 216 & 170 & S 2010-G20Y & 177 & 193 & 176 & D-Y10R & 162 & 137 & 98 \\
\hline S 0530-Y & 253 & 229 & 151 & S 2010-R80B & 176 & 187 & 200 & S 4030-R90B & 83 & 121 & 152 \\
\hline S 0530-Y10R & 255 & 222 & 148 & S 2010-Y30R & 212 & 192 & 165 & S 4050-Y80R & 143 & 58 & 45 \\
\hline S 0550-Y10R & 255 & 213 & 100 & S 2020-Y70R ${ }^{1}$ & 213 & 165 & 149 & S 4550-Y80R & 130 & 45 & 35 \\
\hline S 0550-Y40R & 255 & 169 & 100 & S 2040-Y10R & 213 & 173 & 95 & S 5010-G90Y & 136 & 129 & 107 \\
\hline S 0550-Y70R & 255 & 152 & 118 & S 2060-Y10R ${ }^{1}$ & 205 & 142 & 51 & -Y70R & 147 & 102 & 88 \\
\hline S 0570-Y50R & 253 & 131 & 55 & S 2570-Y30R & 197 & 114 & 0 & S 5030-Y40R & 142 & 94 & 58 \\
\hline S 1002 & 222 & 220 & 210 & S 3005-R80B & 165 & 172 & 178 & S 5502-Y & 123 & 121 & 114 \\
\hline S 1005-R20B & 223 & 213 & 215 & S 3005-Y80R & 183 & 169 & 163 & S 6010-Y10R & 119 & 104 & 83 \\
\hline S 1005-R80B & 214 & 219 & 225 & S 3010-B30G & 147 & 170 & 173 & S 6500-N & 102 & 102 & 101 \\
\hline S 1005-Y & 228 & 224 & 203 & S 3010-G60Y & 169 & 172 & 148 & S 7010-Y70R & 93 & 69 & 61 \\
\hline S 1005-Y50R & 230 & 217 & 202 & S 3010-Y & 181 & 170 & 141 & & & & \\
\hline S 1010-B30G & 196 & 219 & 221 & S 3010-Y50R & 189 & 164 & 146 & & & & \\
\hline
\end{tabular}

${ }^{1}$ Additional colours for padding to total 70 ; NCS code approximate

\section{Appendix B. Colorimetric vs. Photographic Comparison for Image Capture Verification}

To assess the accuracy of the colour calibration we compared image captures of the Colourchecker and NCS exterior set with colorimetric readings under D65 illuminant. Averaged pixel values were extracted from the images in $L^{*} a^{*} b^{*}$ space, and their deviations to the colorimetric reference expressed as CIE $\Delta E$.

Deviations for the 24 classic patches of a Colourchecker Passport (issue 0616) and for a subset of the NCS exterior set are collated in Tables A2 and A3, respectively. The 51 colours selected for verification from the NCS exterior set are grouped according to chromaticness, blackness, and hue, with similarly grouped average deviations. 
Table A2. Colour differences $\Delta E$ between colour-calibrated image of Colourchecker patches and their colorimetric values to verify the image capture and colour calibration workflow.

\begin{tabular}{|c|c|c|c|c|c|c|c|c|}
\hline \multicolumn{2}{|c|}{$\begin{array}{c}\text { Colourchecker } \\
\text { Patch }\end{array}$} & \multicolumn{3}{|c|}{ Colorimetric (D65) } & \multicolumn{3}{|c|}{ Image Capture } & \multirow{2}{*}{$\Delta \mathrm{E}_{2000}$} \\
\hline Index & Name & $\mathbf{L}$ & ${ }^{*} \mathbf{a}$ & *b & $\mathrm{L}$ & *a & *b & \\
\hline 1 & Dark skin & 39.0 & 10.4 & 12.5 & 39.7 & 11.3 & 13.0 & 0.8 \\
\hline 2 & Light skin & 64.6 & 17.1 & 17.1 & 67.5 & 14.5 & 14.0 & 2.9 \\
\hline 3 & Blue sky & 50.8 & -0.8 & -21.5 & 51.1 & 1.5 & -22.5 & 2.1 \\
\hline 4 & Foliage & 43.3 & -14.7 & 21.5 & 44.4 & -16.2 & 22.4 & 1.2 \\
\hline 5 & Blue flower & 56.3 & 12.8 & -24.7 & 55.4 & 15.0 & -26.0 & 2.3 \\
\hline 6 & Bluish green & 72.3 & -31.0 & -0.3 & 71.8 & -31.7 & 0.2 & 0.8 \\
\hline 7 & Orange & 63.5 & 33.8 & 53.4 & 63.7 & 29.9 & 51.9 & 1.9 \\
\hline 8 & Purplish blue & 40.8 & 18.5 & -46.0 & 42.1 & 24.2 & -47.8 & 5.5 \\
\hline 9 & Moderate red & 52.7 & 45.9 & 11.6 & 51.8 & 46.5 & 12.6 & 0.7 \\
\hline 10 & Purple & 31.2 & 23.4 & -24.5 & 32.7 & 24.0 & -21.3 & 1.1 \\
\hline 11 & Yellow green & 73.0 & -28.3 & 56.9 & 73.7 & -32.3 & 59.2 & 0.5 \\
\hline 12 & Orange Yellow & 72.6 & 15.1 & 67.5 & 72.4 & 11.2 & 68.1 & 2.4 \\
\hline 13 & Blue & 29.2 & 23.4 & -48.9 & 29.9 & 29.4 & -49.9 & 2.9 \\
\hline 14 & Green & 56.0 & -41.3 & 32.5 & 56.7 & -43.8 & 34.2 & 1.0 \\
\hline 15 & Red & 42.7 & 48.2 & 24.5 & 44.9 & 48.2 & 20.9 & 3.4 \\
\hline 16 & Yellow & 83.3 & -2.7 & 82.2 & 82.7 & -7.4 & 83.1 & 1.9 \\
\hline 17 & Magenta & 52.7 & 50.4 & -18.1 & 53.1 & 52.4 & -15.4 & 1.8 \\
\hline 18 & Cyan & 51.7 & -23.6 & -26.5 & 52.1 & -17.6 & -27.5 & 1.2 \\
\hline 19 & White & 97.0 & -1.1 & 2.8 & 97.0 & 0.0 & 0.0 & 3.0 \\
\hline 20 & Neutral 8 & 81.4 & -0.7 & -0.2 & 82.8 & -1.4 & -0.3 & 1.3 \\
\hline 21 & Neutral 6.5 & 67.8 & -1.1 & -0.6 & 68.8 & -0.9 & -1.1 & 0.9 \\
\hline 22 & Neutral 5 & 50.6 & -0.3 & -0.2 & 52.2 & -0.5 & -0.1 & 1.6 \\
\hline 23 & Neutral 3.5 & 37.3 & -0.3 & -1.4 & 37.8 & -0.4 & -0.5 & 1.0 \\
\hline 24 & Black & 19.9 & 0.2 & -0.7 & 23.1 & 0.1 & -0.8 & 2.2 \\
\hline & & & & & & & Avg & 1.8 \\
\hline & & & & & & & Max & 5.5 \\
\hline
\end{tabular}

Table A3. Colour differences $\Delta E$ between colour-calibrated images and colorimetric values of 51 representative colours from the NCS exterior set to verify the image capture and colour calibration workflow. The colours are grouped according to chromaticness, blackness, and hue. See Figure 4 for a sample capture containing some of these colours.

\begin{tabular}{|c|c|c|c|c|c|c|c|c|c|c|}
\hline \multirow{3}{*}{ Hue } & \multicolumn{9}{|c|}{ Chromaticness } & \multirow{3}{*}{$\begin{array}{l}\text { Hue } \\
\text { Avg }\end{array}$} \\
\hline & \multicolumn{3}{|c|}{0 (Neutral) } & \multicolumn{3}{|c|}{$2-10$ (Low) } & \multicolumn{3}{|c|}{ 20-30 (Moderate) } & \\
\hline & $\begin{array}{c}\text { Blackness } \\
5-15\end{array}$ & \begin{tabular}{|c} 
Blackness \\
$20-40$
\end{tabular} & \begin{tabular}{|c} 
Blackness \\
$50-70$
\end{tabular} & $\begin{array}{c}\text { Blackness } \\
10-15\end{array}$ & $\begin{array}{c}\text { Blackness } \\
20-40\end{array}$ & \begin{tabular}{|c} 
Blackness \\
$50-60$
\end{tabular} & \begin{tabular}{|c|} 
Blackness \\
$20-30$
\end{tabular} & $\begin{array}{c}\text { Blackness } \\
40-50\end{array}$ & $\begin{array}{c}\text { Blackness } \\
60-70\end{array}$ & \\
\hline $\mathrm{Y}$ & \multirow{9}{*}{4.8} & \multirow{9}{*}{2.9} & \multirow{9}{*}{3.6} & 6.4 & 4.1 & 3.5 & \begin{tabular}{|l|}
2.0 \\
\end{tabular} & 1.7 & 0.4 & 3.0 \\
\hline Y/R & & & & 3.7 & 3.9 & 1.8 & 0.4 & 5.7 & 2.2 & 2.9 \\
\hline $\mathrm{R}$ & & & & 4.3 & 2.8 & 2.1 & 2.5 & 2.7 & 2.6 & 2.8 \\
\hline $\mathrm{R} / \mathrm{B}$ & & & & 4.2 & 2.2 & 2.7 & 4.0 & 2.9 & 1.9 & 3.0 \\
\hline B & & & & 4.5 & 3.0 & 3.3 & 3.8 & 3.5 & 3.9 & 3.6 \\
\hline $\mathrm{B} / \mathrm{G}$ & & & & 4.6 & 3.4 & 3.1 & 3.5 & 2.6 & 2.8 & 3.3 \\
\hline G & & & & 2.8 & 4.4 & 2.8 & 1.4 & 1.9 & 2.8 & 2.7 \\
\hline $\mathrm{G} / \mathrm{Y}$ & & & & 3.2 & 3.1 & 2.3 & 1.2 & 1.9 & 0.9 & 2.1 \\
\hline $\begin{array}{c}\text { Blackn. } \\
\text { Avg }\end{array}$ & & & & 4.2 & 3.4 & 2.7 & 2.3 & 2.9 & 2.2 & \\
\hline $\begin{array}{c}\text { Chrom. } \\
\text { Avg }\end{array}$ & \multicolumn{3}{|c|}{3.7} & \multicolumn{3}{|c|}{3.4} & \multicolumn{3}{|c|}{2.5} & \\
\hline
\end{tabular}




\section{Appendix C. Computed Match Histograms}
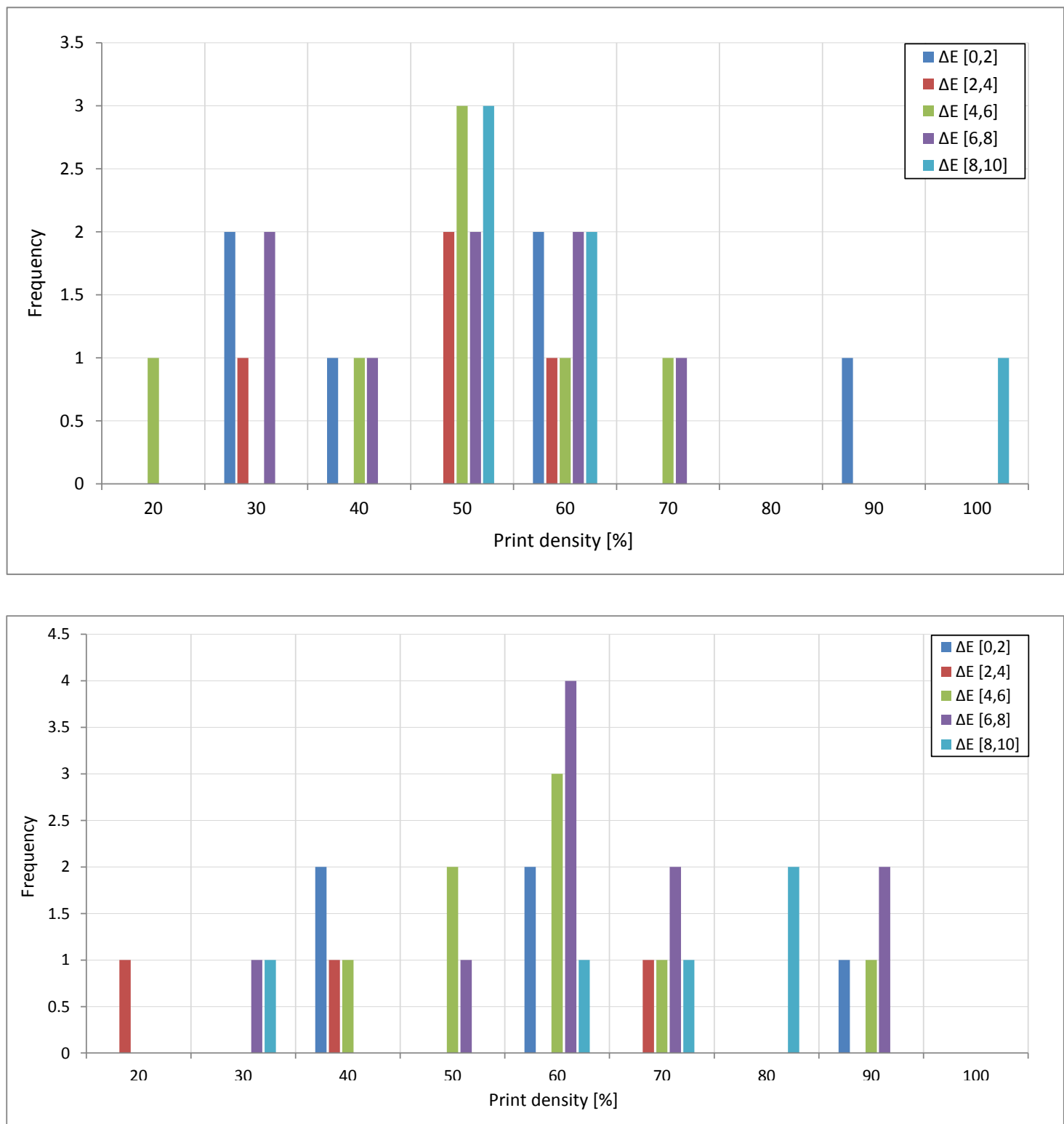

Figure A1. Histograms of computationally matched printed glass samples in Table 1 with ink volumes of 10 (top) and 20 (bottom) pl. Each histogram is itemised for print densities of $20-100 \%$ (no matches were found for $10 \%)$, and for $\Delta E$ per print density. 


\section{Appendix D. Visual Matches}

Table A4. Results from our visual matching experiment for the red-brown colour group. A visual average colour $\mu_{v}$ was obtained from the matched NCS exterior set samples, weighted by their frequency. The weighted population standard deviation $\sigma_{v}$ was then derived based on each samples' colour difference $\Delta E$ to $\mu_{v}$. The computationally matched NCS exterior set samples are highlighted in red.

\begin{tabular}{|c|c|c|c|c|c|c|c|c|c|c|c|c|}
\hline \multirow{2}{*}{$\begin{array}{l}\text { Glass } \\
\text { Label }\end{array}$} & \multicolumn{12}{|c|}{ Visual Matches } \\
\hline & $\begin{array}{c}\text { Card } \\
\text { Label }\end{array}$ & Freq & Weight & NCS Code & $\mathbf{L}^{*}$ & a* & b* & $\mu_{v}\left(L^{*}\right)$ & $\mu_{v}\left(a^{*}\right)$ & $\mu_{v}\left(b^{*}\right)$ & $\Delta E\left(\mu_{v}\right)$ & $\sigma_{\mathrm{v}}(\Delta \mathrm{E})$ \\
\hline \multirow{8}{*}{$\mathbf{A}$} & 380 & 21 & 0.48 & 7020 Y50R & 28.79 & 12.54 & 19.97 & \multirow{8}{*}{32.09} & \multirow{8}{*}{14.71} & \multirow{8}{*}{20.26} & 2.91 & \multirow{8}{*}{4.82} \\
\hline & 326 & 8 & 0.18 & 6030 Y50R & 36.16 & 16.30 & 26.24 & & & & 4.50 & \\
\hline & 406 & 7 & 0.16 & 6030 Y70R & 32.96 & 18.41 & 18.04 & & & & 4.06 & \\
\hline & 333 & 3 & 0.07 & 7020 Y70R & 26.25 & 15.83 & 16.23 & & & & 4.51 & \\
\hline & 350 & 2 & 0.05 & 6020 Y70R & 39.72 & 14.05 & 15.40 & & & & 12.89 & \\
\hline & 335 & 1 & 0.02 & 5030 Y60R & 46.77 & 15.94 & 18.37 & & & & 6.86 & \\
\hline & 351 & 1 & 0.02 & 6020 Y50R & 41.14 & 11.10 & 19.18 & & & & 7.57 & \\
\hline & 395 & 1 & 0.02 & $5040 Y 70 R$ & 41.18 & 21.90 & 18.94 & & & & 8.36 & \\
\hline \multirow{7}{*}{ B } & 353 & 28 & 0.64 & $6020-Y 10 R$ & 43.53 & 3.94 & 21.65 & \multirow{7}{*}{45.05} & \multirow{7}{*}{4.01} & \multirow{7}{*}{22.18} & 1.45 & \multirow{7}{*}{5.31} \\
\hline & 355 & 9 & 0.20 & 5020-Y10R & 52.02 & 2.79 & 21.67 & & & & 6.98 & \\
\hline & 341 & 3 & 0.07 & $6030-Y 10 R$ & 39.62 & 5.41 & 32.07 & & & & 6.37 & \\
\hline & 327 & 1 & 0.02 & 7010-Y10R & 38.99 & 2.11 & 13.25 & & & & 7.62 & \\
\hline & 399 & 1 & 0.02 & $7020-Y 10 R$ & 30.31 & 4.54 & 23.04 & & & & 12.56 & \\
\hline & 335 & 1 & 0.02 & 5030-Y60R & 46.77 & 15.94 & 18.37 & & & & 13.44 & \\
\hline & 397 & 1 & 0.02 & $4020-Y 10 R$ & 60.18 & 2.35 & 24.01 & & & & 15.06 & \\
\hline \multirow{4}{*}{ C } & 332 & 31 & 0.89 & 6030-Y30R & 38.98 & 9.29 & 28.86 & \multirow{4}{*}{40.60} & \multirow{4}{*}{9.67} & \multirow{4}{*}{29.03} & 1.49 & \multirow{4}{*}{4.62} \\
\hline & 348 & 2 & 0.06 & 4040-Y30R & 53.84 & 14.08 & 33.51 & & & & 13.40 & \\
\hline & 393 & 1 & 0.03 & 5030-Y30R & 48.20 & 10.77 & 25.65 & & & & 7.50 & \\
\hline & 392 & 1 & 0.03 & 4030-Y30R & 56.91 & 11.64 & 28.84 & & & & 16.25 & \\
\hline \multirow{4}{*}{ D } & 327 & 25 & 0.64 & 7010-Y10R & 38.99 & 2.11 & 13.25 & & & & 1.95 & \\
\hline & 354 & 7 & 0.18 & 6010-Y10R & 47.13 & 1.89 & 14.47 & & & & 5.61 & \\
\hline & 379 & 5 & 0.13 & 7010-Y30R & 37.99 & 4.30 & 13.13 & 41.18 & 2.29 & 13.51 & 3.81 & 4.57 \\
\hline & 398 & 2 & 0.05 & 5010-Y10R & 55.75 & 0.98 & 14.44 & & & & 14.58 & \\
\hline & 403 & 28 & 0.68 & 7010-Y90R & 32.30 & 9.63 & 7.01 & & & & 0.84 & \\
\hline & 381 & 8 & 0.20 & 7010-Y70R & 31.19 & 8.37 & 10.26 & & & & 2.66 & \\
\hline $\mathbf{E}$ & 404 & 3 & 0.07 & $6010-Y 90 R$ & 44.70 & 8.95 & 6.25 & 32.98 & 9.27 & 7.70 & 10.19 & 3.92 \\
\hline & 375 & 1 & 0.02 & 8010-Y90R & 18.88 & 9.90 & 7.06 & & & & 10.44 & \\
\hline & 377 & 1 & 0.02 & 6010-Y50R & 45.34 & 6.54 & 11.48 & & & & 11.54 & \\
\hline & 333 & 16 & 0.41 & $7020-Y 70 R$ & 26.25 & 15.83 & 16.23 & & & & 3.24 & \\
\hline & 381 & 12 & 0.31 & 7010-Y70R & 31.19 & 8.37 & 10.26 & & & & 4.81 & \\
\hline & 388 & 4 & 0.10 & $7020-Y 90 R$ & 23.31 & 16.39 & 11.17 & & & & 4.55 & \\
\hline $\mathbf{F}$ & 350 & 2 & 0.05 & 6020-Y70R & 39.72 & 14.05 & 15.40 & 29.23 & 13.41 & 13.30 & 8.64 & 4.73 \\
\hline & 406 & 2 & 0.05 & $6030-Y 70 R$ & 32.96 & 18.41 & 18.04 & & & & 4.55 & \\
\hline & 402 & 2 & 0.05 & $6020-Y 90 R$ & 37.35 & 14.57 & 8.64 & & & & 7.57 & \\
\hline & 403 & 1 & 0.03 & 7010-Y90R & 32.30 & 9.63 & 7.01 & & & & 6.08 & \\
\hline & 339 & 21 & 0.50 & 6020-Y30R & 42.51 & 6.69 & 20.61 & & & & 0.81 & \\
\hline & 379 & 7 & 0.17 & 7010-Y30R & 37.99 & 4.30 & 13.13 & & & & 5.20 & \\
\hline & 353 & 6 & 0.14 & 6020-Y10R & 43.53 & 3.94 & 21.65 & & & & 3.49 & \\
\hline & 390 & 3 & 0.07 & 5020-Y30R & 50.64 & 7.58 & 20.99 & & & & 8.61 & \\
\hline G & 378 & 2 & 0.05 & 7020-Y30R & 30.46 & 8.95 & 22.47 & 41.72 & 6.22 & 19.77 & 10.06 & 4.61 \\
\hline & 351 & 1 & 0.02 & 6020-Y50R & 41.14 & 11.10 & 19.18 & & & & 3.45 & \\
\hline & 393 & 1 & 0.02 & 5030-Y30R & 48.20 & 10.77 & 25.65 & & & & 8.67 & \\
\hline & 399 & 1 & 0.02 & $7020-Y 10 R$ & 30.31 & 4.54 & 23.04 & & & & 10.24 & \\
\hline & 382 & 16 & 0.33 & 7010-Y50R & 36.85 & 7.23 & 12.53 & & & & 3.01 & \\
\hline & 381 & 16 & 0.33 & 7010-Y70R & 31.19 & 8.37 & 10.26 & & & & 2.44 & \\
\hline H & 329 & 8 & 0.17 & $8010-Y 50 R$ & 23.49 & 6.77 & 12.59 & 33.23 & 7.14 & 11.81 & 7.42 & 4.71 \\
\hline & 379 & 6 & 0.13 & 7010-Y30R & 37.99 & 4.30 & 13.13 & & & & 5.21 & \\
\hline & 377 & 2 & 0.04 & $6010-Y 50 R$ & 45.34 & 6.54 & 11.48 & & & & 10.56 & \\
\hline
\end{tabular}


Table A5. Results from our visual matching experiment for the green-blue colour group. See Table A4 for an explanation of the data.

\begin{tabular}{|c|c|c|c|c|c|c|c|c|c|c|c|c|}
\hline \multirow{2}{*}{$\begin{array}{l}\text { Glass } \\
\text { Label }\end{array}$} & \multicolumn{12}{|c|}{ Visual Matches } \\
\hline & $\begin{array}{l}\text { Card } \\
\text { Label }\end{array}$ & Freq & Weight & NCS Code & L* & a* & b* & $\mu_{v}\left(L^{*}\right)$ & $\mu_{v}\left(a^{*}\right)$ & $\mu_{v}(b *)$ & $\Delta E\left(\mu_{v}\right)$ & $\sigma_{\mathrm{v}}(\Delta \mathrm{E})$ \\
\hline \multirow{10}{*}{ I } & 373 & 13 & 0.33 & 6010 G10Y & 44.40 & -9.32 & 3.68 & \multirow{10}{*}{43.87} & \multirow{10}{*}{-10.13} & \multirow{10}{*}{6.90} & 2.67 & \multirow{10}{*}{6.53} \\
\hline & 328 & 11 & 0.28 & $7010 \mathrm{GlOY}$ & 36.49 & -9.82 & 4.00 & & & & 6.94 & \\
\hline & 346 & 8 & 0.20 & $5020 \mathrm{G} 30 \mathrm{Y}$ & 51.95 & -12.30 & 12.51 & & & & 8.48 & \\
\hline & 336 & 2 & 0.05 & $7010 \mathrm{G} 50 \mathrm{Y}$ & 37.54 & -5.98 & 9.95 & & & & 7.72 & \\
\hline & 374 & 1 & 0.03 & $6010 \mathrm{G} 50 \mathrm{Y}$ & 46.74 & -5.80 & 10.23 & & & & 5.97 & \\
\hline & 334 & 1 & 0.03 & $6020 \mathrm{GlOY}$ & 39.65 & -18.31 & 9.25 & & & & 6.06 & \\
\hline & 337 & 1 & 0.03 & $5020 \mathrm{GlOY}$ & 49.91 & -14.74 & 6.77 & & & & 6.45 & \\
\hline & 331 & 1 & 0.03 & $6020 \mathrm{G} 50 \mathrm{Y}$ & 43.58 & -9.30 & 17.29 & & & & 8.32 & \\
\hline & 365 & 1 & 0.03 & $5010 \mathrm{GlOY}$ & 53.33 & -9.24 & 4.60 & & & & 9.53 & \\
\hline & 370 & 1 & 0.03 & $5020 \mathrm{G} 50 \mathrm{Y}$ & 52.15 & -8.11 & 16.10 & & & & 10.79 & \\
\hline \multirow{5}{*}{ J } & 328 & 22 & 0.56 & $7010-\mathrm{G} 10 \mathrm{Y}$ & 36.05 & -10.00 & 4.18 & \multirow{5}{*}{40.42} & \multirow{5}{*}{-11.60} & \multirow{5}{*}{5.13} & 4.09 & \multirow{5}{*}{5.81} \\
\hline & 337 & 7 & 0.18 & 5020-G10Y & 49.84 & -14.78 & 6.81 & & & & 9.25 & \\
\hline & 373 & 5 & 0.13 & 6010-G10Y & 44.44 & -9.33 & 3.83 & & & & 4.34 & \\
\hline & 334 & 4 & 0.10 & 6020-G10Y & 39.69 & -18.29 & 9.16 & & & & 4.73 & \\
\hline & 365 & 1 & 0.03 & 5010-G10Y & 53.45 & -9.25 & 4.61 & & & & 12.89 & \\
\hline \multirow{6}{*}{$\mathbf{K}$} & 336 & 20 & 0.48 & $7010-G 50 Y$ & 37.54 & -5.98 & 9.95 & \multirow{6}{*}{42.79} & \multirow{6}{*}{-6.89} & \multirow{6}{*}{12.00} & 4.85 & \\
\hline & 374 & 8 & 0.19 & $6010-G 50 Y$ & 46.74 & -5.80 & 10.23 & & & & 4.00 & \\
\hline & 331 & 7 & 0.17 & 6020-G50Y & 43.58 & -9.30 & 17.29 & & & & 3.72 & \\
\hline & 370 & 5 & 0.12 & 5020-G50Y & 52.15 & -8.11 & 16.10 & & & & 9.47 & 5.73 \\
\hline & 346 & 1 & 0.02 & 5020-G30Y & 51.95 & -12.30 & 12.51 & & & & 9.46 & \\
\hline & 371 & 1 & 0.02 & 5010-G50Y & 54.79 & -5.42 & 8.94 & & & & 12.17 & \\
\hline & 362 & 18 & 0.40 & 7010-B30G & 33.15 & -6.37 & -3.55 & & & & 2.68 & \\
\hline & 384 & 16 & 0.36 & 7010-B70G & 32.56 & -9.70 & -0.86 & & & & 2.13 & \\
\hline & 328 & 3 & 0.07 & 7010-G10Y & 36.49 & -9.82 & 4.00 & & & & 5.24 & \\
\hline & 394 & 2 & 0.04 & 6010-B30G & 45.60 & -6.38 & -4.29 & & & & 10.58 & \\
\hline $\mathbf{L}$ & 386 & 2 & 0.04 & 6010-B70G & 44.42 & -9.17 & -1.52 & 34.12 & -8.17 & -1.60 & 9.01 & 4.84 \\
\hline & 330 & 1 & 0.02 & 7020-B30G & 25.52 & -11.63 & -7.74 & & & & 8.42 & \\
\hline & 373 & 1 & 0.02 & 6010-G10Y & 44.44 & -9.33 & 3.83 & & & & 10.05 & \\
\hline & 336 & 1 & 0.02 & 7010-G50Y & 37.54 & -5.98 & 9.95 & & & & 10.20 & \\
\hline & 338 & 1 & 0.02 & 8010-B70G & 20.66 & -10.36 & -0.83 & & & & 10.36 & \\
\hline & 336 & 28 & 0.72 & $7010-G 50 Y$ & 37.54 & -5.98 & 9.95 & & & & 2.43 & \\
\hline $\mathbf{M}$ & 374 & 10 & 0.26 & 6010-G50Y & 46.74 & -5.80 & 10.23 & 40.34 & -5.92 & 10.00 & 5.93 & 4.29 \\
\hline & 371 & 1 & 0.03 & 5010-G50Y & 54.79 & -5.42 & 8.94 & & & & 14.23 & \\
\hline & 328 & 20 & 0.45 & $7010-G 10 Y$ & 36.49 & -9.82 & 4.00 & & & & 3.31 & \\
\hline & 373 & 14 & 0.32 & 6010-G10Y & 44.40 & -9.32 & 3.68 & & & & 3.75 & \\
\hline & 384 & 4 & 0.09 & 7010-B70G & 32.56 & -9.70 & -0.86 & & & & 7.54 & \\
\hline & 337 & 2 & 0.05 & 5020-G10Y & 49.91 & -14.74 & 6.77 & & & & 9.94 & \\
\hline $\mathbf{N}$ & 344 & 1 & 0.02 & 5020-B90G & 50.02 & -15.32 & 1.96 & 40.33 & -9.95 & 3.88 & 10.21 & 5.47 \\
\hline & 365 & 1 & 0.02 & 5010-G10Y & 53.33 & -9.24 & 4.60 & & & & 12.69 & \\
\hline & 346 & 1 & 0.02 & 5020-G30Y & 51.95 & -12.30 & 12.51 & & & & 12.88 & \\
\hline & 336 & 1 & 0.02 & 7010-G50Y & 37.54 & -5.98 & 9.95 & & & & 6.90 & \\
\hline & 383 & 19 & 0.48 & 6020-B30G & 38.85 & -12.18 & -8.56 & & & & 4.95 & \\
\hline & 330 & 14 & 0.35 & 7020-B30G & 25.52 & -11.63 & -7.74 & & & & 5.70 & \\
\hline O & 361 & 5 & 0.13 & 6030-B30G & 30.82 & -17.74 & -11.37 & 32.90 & -12.39 & -8.37 & 3.85 & 5.18 \\
\hline & 362 & 2 & 0.05 & 7010-B30G & 33.15 & -6.37 & -3.55 & & & & 6.25 & \\
\hline & 384 & 24 & 0.59 & 7010-B70G & 32.56 & -9.70 & -0.86 & & & & 2.82 & \\
\hline & 385 & 9 & 0.22 & 6020-B70G & 39.50 & -16.74 & -2.46 & & & & 5.43 & \\
\hline & 386 & 3 & 0.07 & 6010-B70G & 44.42 & -9.17 & -1.52 & & & & 8.03 & \\
\hline $\mathbf{P}$ & 362 & 2 & 0.05 & 7010-B30G & 33.15 & -6.37 & -3.55 & 35.57 & -11.30 & -1.42 & 5.14 & 5.16 \\
\hline & 340 & 1 & 0.02 & 7020-B70G & 26.08 & -17.70 & -1.38 & & & & 9.05 & \\
\hline & 394 & 1 & 0.02 & 6010-B30G & 45.60 & -6.38 & -4.29 & & & & 10.27 & \\
\hline & 344 & 1 & 0.02 & 5020-B90G & 50.02 & -15.32 & 1.96 & & & & 14.16 & \\
\hline
\end{tabular}

\section{References}

1. Eidgenössische Kommission für Denkmalpflege. Energie und Baudenkmal_Empfehlungen für die Energetische Verbesserung von Baudenkmälern; Technical Report; Bundesamt für Energie: Bern, Switzerland, 2009. Available online: https://www.admin.ch/gov/de/start/dokumentation/medienmitteilungen.msg-id28129.html (accessed on 9 August 2017).

2. Lee, K.T.; Lee, J.Y.; Seo, S.; Guo, L.J. Colored ultrathin hybrid photovoltaics with high quantum efficiency. Light Sci. Appl. 2014, 3, e215. doi:10.1038/1sa.2014.96.

3. Escarré, J.; Li, H.Y.; Sansonnens, L.; Galliano, F.; Cattaneo, G.; Heinstein, P.; Nicolay, S.; Bailat, J.; Eberhard, S.; Ballif, C.; et al. When PV modules are becoming real building elements: White solar module, a revolution for BIPV. In Proceedings of the 42nd Photovoltaic Specialist Conference (PVSC), New Orleans, LA, USA, 4-19 June 2015. doi:10.1109/PVSC.2015.7355630.

4. Wittkopf, S. Coloured Photovoltaics. In Proceedings of the Advanced Building Skins, Bern, Switzerland, October 2016; doi:10.5281/zenodo.399209. Available online: http://zenodo.org/record/399209 (accessed on 16 August 2017).

5. Green, M.A.; Emery, K.; Hishikawa, Y.; Warta, W.; Dunlop, E.D. Solar cell efficiency tables (Version 45). Prog. Photovolt. Res. Appl. 2015, 23, 1-9. doi:10.1002/pip.2573. 
6. Manwiller, R. Digital Ceramic Printing. In Advances in Porcelain Enamel Technology; John Wiley \& Sons, Inc.: Hoboken, NJ, USA, 2010; pp. 155-156. doi:10.1002/9780470640906.ch29.

7. Keith Emery. Photovoltaic Efficiency Measurements. Available online: http://www.nrel.gov/docs/gen/ fy04/368311.pdf (accessed on 16 August 2017).

8. Hård, A.; Sivik, L.; Tonnquist, G. NCS, Natural Color System-From Concept to Research and Applications. Part I. Color Res. Appl. 1996, 21, 180-205. doi:10.1002/(SICI)1520-6378(199606)21:3<180::AID-COL2>3.0.CO;2-O.

9. Sharma, G.; Wu, W.; Dalal, E.N. The CIEDE2000 color-difference formula: Implementation notes, supplementary test data, and mathematical observations. Color Res. Appl. 2005, 30, 21-30. doi:10.1002/col.20070.

10. Rehsteiner, J.; Wettstein, L.; Stefanie, S. (Eds.) FARBRAUM STADT: BOX ZRH Das Buch; Haus der Farbe: Zürich, Switzerland, 2010. Available online: http:/ /www.farbraumstadt.ch (accessed on 16 August 2017).

11. Enberg, K. NCS Exterior Design Tool Guide; NCS Colour AB: Stockholm, Sweden, 2010. Available online: http:/ /ncscolour.com/product/ncs-exterior/ (accessed on 16 August 2017).

12. Gill, G. Argyll Colour Management System. Available online: https://www.argyllcms.com (accessed on 16 August 2017).

13. Pascale, D. RGB Coordinates of the Macbeth ColorChecker. Available online: http://www.babelcolor.com/ index_htm_files/RGBCoordinatesoftheMacbethColorChecker.pdf (accessed on 16 August 2017).

14. RawTherapee Development Team. Available online: http:// rawtherapee.com/ (accessed on 16 August 2017).

15. Schneider, C.A.; Rasband, W.S.; Eliceiri, K.W. NIH Image to ImageJ: 25 years of image analysis. Nat. Methods 2012, 9, 671-675. doi:10.1038/nmeth.2089.

16. Rasband, W. ImageJ-A Java iMage Processing Program Inspired by NIH Image. Available online: https: / imagej.nih.gov/ij/ (accessed on 16 August 2017).

17. Barilla, M.E. ImageJ Colour Transformer Plugin. Available online: https://imagej.nih.gov/ij/plugins/colortransforms.html (accessed on 16 August 2017).

18. Colormine.org. CIE2000 Calculator. Available online: http://colormine.org/delta-e-calculator/cie2000 (accessed on 16 August 2017).

19. Chou, T.R.; Chang, S.K. Color Calibration of Recovering High Dynamic Range Images. In Proceedings of the 2008 International Conference on Computer Science and Software Engineering, Wuhan, China, 12-14 December 2008; pp. 286-289. doi:10.1109/CSSE.2008.1350.

20. Varghese, D.; Wanat, R.; Mantiuk, R.K. Colorimetric Calibration of High Dynamic Range Images with a ColorChecker Chart. In Proceedings of the HDRi 2014-Second International Conference and SME Workshop on HDR Imaging, Sarajevo, Bosnia and Herzegovina, 4-5 March 2014. Available online: http:/ / www.cl.cam.ac.uk/ rkm38/pdfs/varghese14color_calib.pdf (accessed on 16 August 2017).

21. NumPy Developers. NumPy: Scientific Computing with Python. Available online: http:/ /www.numpy.org/ (accessed on 16 August 2017).

22. Taylor, G. Python Colormath Module. Available online: https://pypi.python.org/pypi/colormath/ (accessed on 16 August 2017).

23. McNamara, J. XlsxWriter 0.9.6: A Python Module for Creating Excel XLSX Files. Available online: https:/ / pypi.python.org/pypi/XlsxWriter (accessed on 16 August 2017).

24. Abramov, I.; Gordon, J.; Feldman, O.; Chavarga, A. Sex and vision II: Color appearance of monochromatic lights. Biol. Sex Differ. 2012, 3, 21. doi:10.1186/2042-6410-3-21.

25. Mokrzycki, W.S.; Tatol, M. Color difference $\Delta$ E: A Survey. Mach. Gr. Vis. 2011, $20,383-411$. Available online: www.researchgate.net/publication/236023905_Color_difference_Delta_E_-_A_survey (accessed on 16 August 2017).

(c) 2017 by the authors. Licensee MDPI, Basel, Switzerland. This article is an open access article distributed under the terms and conditions of the Creative Commons Attribution (CC BY) license (http:/ / creativecommons.org/licenses/by/4.0/). 